\title{
Marine Oil Supplements for Arthritis Pain: A Systematic Review and Meta-Analysis of Randomized Trials
}

\author{
Ninna K. Senftleber ${ }^{1,2,+}$, Sabrina M. Nielsen ${ }^{1,2,+}$, Jens R. Andersen ${ }^{2}$, Henning Bliddal ${ }^{1}$, \\ Simon Tarp ${ }^{1}$, Lotte Lauritzen ${ }^{2}$, Daniel E. Furst ${ }^{3}$, Maria E. Suarez-Almazor ${ }^{4}$, Anne Lyddiatt ${ }^{5}$ and \\ Robin Christensen ${ }^{1, *}$ \\ 1 Musculoskeletal Statistics Unit, The Parker Institute, Bispebjerg and Frederiksberg Hospital, \\ 2000 Copenhagen F, Denmark; ninnaks@hotmail.com (N.K.S.); sabrina.mai.nielsen@regionh.dk (S.M.N.); \\ henning.bliddal@regionh.dk (H.B.); simon.tarp@regionh.dk (S.T.) \\ 2 Department of Nutrition, Exercise and Sports, University of Copenhagen, 1958 FC Copenhagen, Denmark; \\ jra@nexs.ku.dk (J.R.A.); 11@nexs.ku.dk (L.L.) \\ 3 Division of Rheumatology, University of California, Los Angeles, CA 90095, USA; defurst@mednet.ucla.edu \\ 4 The University of Texas MD Anderson Cancer Center, Houston, TX 77030, USA; \\ msalmazor@mdanderson.org \\ 5 Musculoskeletal Group, Cochrane Collaboration, Ottawa, ON K1H 8L6, Canada; lyddiatt@lyddiatt.ca \\ * Correspondence: robin.christensen@regionh.dk; Tel.: +45-3816-4165 \\ + These authors contributed equally to this work.
}

Received: 20 October 2016; Accepted: 21 December 2016; Published: 6 January 2017

\begin{abstract}
Arthritis patients often take fish oil supplements to alleviate symptoms, but limited evidence exists regarding their efficacy. The objective was to evaluate whether marine oil supplements reduce pain and/or improve other clinical outcomes in patients with arthritis. Six databases were searched systematically (24 February 2015). We included randomized trials of oral supplements of all marine oils compared with a control in arthritis patients. The internal validity was assessed using the Cochrane Risk of Bias tool and heterogeneity was explored using restricted maximum of likelihood (REML)-based meta-regression analysis. Grading of Recommendations Assessment, Development and Evaluation (GRADE) was used to rate the overall quality of the evidence. Forty-two trials were included; 30 trials reported complete data on pain. The standardized mean difference (SMD) suggested a favorable effect $(-0.24 ; 95 \%$ confidence interval, $\mathrm{CI},-0.42$ to -0.07 ; heterogeneity, $I^{2}=63 \%$. A significant effect was found in patients with rheumatoid arthritis ( 22 trials; $-0.21 ; 95 \%$ CI, -0.42 to -0.004 ) and other or mixed diagnoses ( 3 trials; $-0.63 ; 95 \% \mathrm{CI},-1.20$ to -0.06 ), but not in osteoarthritis patients ( 5 trials; $-0.17 ; 95 \% \mathrm{CI},-0.57-0.24$ ). The evidence for using marine oil to alleviate pain in arthritis patients was overall of low quality, but of moderate quality in rheumatoid arthritis patients.
\end{abstract}

Keywords: arthritis; marine oil; fish oil; joint pain; rheumatology; complementary medicine; meta-analysis; randomized controlled trials

\section{Introduction}

Arthritis is a musculoskeletal disorder, resulting in joint pain, swelling, stiffness and restricted movement [1,2]. In the period 2010-2012, 22.7\% of American adults reported having medically-diagnosed arthritis [3]. The two most common types of arthritis are rheumatoid arthritis (RA) and osteoarthritis (OA). Osteoarthritis is a heterogeneous, degenerative disease exhibiting inflammatory components, associated with multiple risk factors [4]. In contrast, RA is an autoimmune disease involving higher levels of synovitis (joint inflammation) than OA [2,4]. The extent of synovitis 
has been shown to be associated with pain severity [5,6]. Arthritis patients consider pain the most hampering symptom [7], and the use of nonsteroidal anti-inflammatory drugs (NSAIDs) is substantial across arthritis diagnoses [8,9]. However, NSAIDs are known to cause serious gastrointestinal and occasionally cardiovascular adverse effects [10,11], prompting the search for alternative treatments.

Marine oil is thought to have an analgesic effect in arthritis as a likely consequence of its high content of docosahexaenoic acid (DHA; 22:6n-3) and eicosapentaenoic acid (EPA; 20:5 n-3). Arachidonic acid (AA; 20:4 n-6), as well as DHA and EPA, are used in the production of lipid mediators (e.g., eicosanoids), which are involved (among other functions) in the regulation of inflammation. However, the mediators produced from DHA and EPA shift the balance toward resolution [12]. Thus, supplementation with EPA- and DHA-rich oil could exert an anti-inflammatory effect [13], making it a possible treatment for arthritis pain.

Four meta-analyses have compared fish oil or $n-3$ polyunsaturated fatty acid (PUFA) supplements with control treatment in patients with musculoskeletal complaints [14-17]. Three meta-analyses concluded that such supplements were effective [14-16], whereas the fourth review did not find any effect [17], likely because of different inclusion criteria. In contrast to the previous meta-analyses, our review included a comprehensive search strategy and assessment of the risk of bias and the quality of evidence. Vegetable oils were not included, as these provide $\alpha$-linolenic acid (ALA; 18:3 n-3), from which only marginal amounts of EPA and DHA can be produced in the human body $[18,19]$.

A previous US survey suggested that approximately $90 \%$ of arthritis patients have used, or were using, complementary therapies [20]. Therefore, consumers and health care practitioners need to know whether oral marine oil can reduce arthritis pain.

The aim of this systematic review was to evaluate the effect of marine oil across a broad range of arthritic diseases. Our primary outcome was pain. Secondary outcomes were physical function, inflammation, number of completers (tolerance), withdrawals due to adverse events, and number of serious adverse events (SAEs). Our use of the term "marine oil" refers to all oils of marine origin (e.g., oil from whole fish, seals, and mussels).

\section{Materials}

\subsection{Protocol}

Study selection, assessment of eligibility criteria, data extraction, and statistical analyses were performed based on a predefined protocol registered on PROSPERO (CRD42015016817) following the guidelines from EQUATOR [21] on systematic reviews (PRISMA statement [22]).

\subsection{Data Sources and Searches}

The search was conducted on 24 February 2015 in Medical Literature Analysis and Retrieval System Online (MEDLINE), the Cochrane Library, Excerpta Medica Database (EMBASE), ClinicalTrials.gov, and the World Health Organization International Clinical Trial Registry Platform portal (ICTRP) as recommended by the Cochrane Musculoskeletal Group [23], and in the Web of Science (search strategies are available in Table S1). Reference lists from relevant publications were screened. The initial screening of the records was done by two reviewers (N.K.S. and S.M.N.), and the subsequent assessment on full texts was done independently by the same two reviewers. Disagreements were resolved by consensus or by consulting another reviewer (R.C. or H.B.).

\subsection{Study Selection}

Eligible studies were all randomized controlled trials comparing all types of marine oil supplements (i.e., oils of marine origin such as oil from whole fish, seals, or mussels) with a control treatment applying an add-on design in patients diagnosed with any type of arthritis for a minimum duration of two weeks. No restrictions were applied on age or gender of the participants, dosage, or publication date. Reporting the outcomes of interest was not a criterion for entering the 
systematic review. However, trials included in the main meta-analysis for each outcome had to present complete data (i.e., quantitative data or comprehensive figures), allowing data extraction. Reports with incomplete data for the outcomes of interest were included in a sensitivity analysis using null imputations. Reports had to have at least an abstract written in English, Danish, Swedish, or Norwegian.

\subsection{Data Extraction and Quality Assessment}

The preferred time point of measurements was the last day of the intervention or as late as possible when the participants were still receiving the intervention. According to the guidance document provided by the Cochrane Musculoskeletal Group (aligned with Outcome Measures in Rheumatology [OMERACT]), there is currently no consensus on a generic core outcome measurement instrument set that would apply across all musculoskeletal conditions [23]. However, pain remains a construct of major importance to all (rheumatology) stakeholders (e.g., patients and physicians) [24]. Thus for the purpose of this review we considered pain the primary outcome [25]. For the function construct, we used the most appropriate functional tests (e.g., grip strength was preferred for measuring function in RA, and a walk test was preferred for measuring function in hip or knee OA). We preferred the most commonly used measures of inflammation (e.g., C-reactive protein, CRP, was preferred over erythrocyte sedimentation rate). In case of doubt, we used the outcome chosen by a blinded rheumatologist (H.B.). Authors were contacted in order to obtain data when it was not extractable.

Trials with low internal validity may distort the results from meta-analyses [26], so trials were assessed using the Cochrane Risk of Bias tool [27]. There is evidence that statistically significant outcomes have higher odds of being fully reported [28]; therefore, outcome-reporting bias was assessed using the tool developed by Dwan et al. [29]. Two reviewers (N.K.S. and S.M.N.) independently extracted data and assessed risk of bias. In case of disagreement, consensus was reached by discussion or by consulting a third reviewer (R.C. or S.T.).

\subsection{Data Synthesis and Analysis}

Due to different ways of measuring pain, physical function, and inflammation, treatment effect sizes for each of the studies were expressed as standardized mean differences (SMDs) by dividing the difference in mean values by the pooled standard deviation (SD) for the given outcome. A correction was applied by default by calculating Hedges's $g$ [30] and the variance $\left(\mathrm{SE}^{2}\right)$ was calculated based on the SMD and number of patients in each group [31]. For trials with more than one intervention group, the number of patients in the control group was divided by the number of comparisons, hence avoiding double counting of patients and increasing the standard errors, resulting in more correct estimates. Mean differences at follow-up were used when differences in change from baseline were not available. A negative SMD for pain and inflammation indicates a beneficial (reducing) effect of the intervention, where a positive SMD for physical function indicates a beneficial (increasing) effect. Risk ratios (RRs) were calculated for tolerance, withdrawals due to adverse events, and number of SAEs [23]. The overall SMDs were transformed into a measure that is easier to interpret [23]; the SMD for pain was transformed into average improvement in percentage compared to control [32], assuming an average for pain of $60 \mathrm{~mm}$ on a 100-mm visual analog scale (VAS) with an SD of $20 \mathrm{~mm}$ based on a cohort of arthritis patients [33]. For inflammation, a baseline value of $3.8 \mathrm{mg} / \mathrm{dL}$ CRP and an SD of $5.9 \mathrm{mg} / \mathrm{dL}$ were assumed (equal to the mean baseline value and SD, respectively, of the included studies).

Heterogeneity was investigated using forest plots and the heterogeneity $\left(I^{2}\right)$ statistic $[34,35]$. Random-effects models, based on restricted maximum of likelihood (REML), were used as default option, whereas fixed-effect models were applied as sensitivity analysis.

Sensitivity analyses were conducted by including trials with high risk of outcome-reporting bias and incomplete data on the outcomes of interest by using null imputation, based on the assumption that they did not find any effect. This was done by imputing the value, 0 , for the SMDs, and calculating 
the confidence intervals from the $\mathrm{SE}^{2}$ as usual, i.e., based on the SMD and number of patients in each group [36]. The risk of small-study (e.g., publication) bias across studies was assessed using funnel plots as well as a test for funnel plot asymmetry [37]. Univariate REML-based meta-regression analyses on pain were used to assess the effect of each risk of bias domain and funding source. Analyses were performed using R Software (version 3.2.0) [38].

GRADE (Grading of Recommendations Assessment, Development and Evaluation) was used to rate the overall quality of the evidence based on the assessed risk of bias, publication bias, imprecision, inconsistency, indirectness, and to some degree interpreted this with the apparent magnitude of effect as well [39]. The GRADE ratings reflect the confidence in the results.

\subsection{Additional Analyses}

In order to investigate possible sources of heterogeneity, additional analyses of pain were performed, stratifying the available trials according to trial characteristics (e.g., type of diagnosis) using univariate REML-based meta-regression. Furthermore, a limited number of post hoc analyses were carried out, stratifying for the type of marine oil for pain, and for trial characteristics and risk of bias domains for function and inflammation.

\section{Results}

\subsection{Study Selection}

The search strategy identified 3389 records (Figure 1). Of these, 3212 were excluded because they did not meet the inclusion criteria, and the subsequent full-text assessment resulted in 63 records describing 53 trials eligible for the systematic review. Searching the trial registries ClinicalTrials.gov and the World Health Organization International Clinical Trial Registry Platform portal (ICTRP) contributed an additional nine unique trials for the systematic review; however, none of these trials were published, and no resulting data could be obtained from author contact so they could not be included in the meta-analysis. Review of reference lists of relevant publications identified three potentially relevant trials; one was included [40], and two could not be retrieved [41,42]. In total, 78 records describing 65 trials met the inclusion criteria for the systematic review; of these, 51 records describing 42 trials [40,43-92] were eligible for inclusion in the meta-analyses (reference lists are provided in Section S1). Reasons for exclusion are stated in Figure 1 (reference lists are provided in Sections S2 and S3). One trial was ongoing at the time of publication [93], and we received no answer after contacting the author. Authors of 20 trials were contacted for additional information; four $[46,72,89,90]$ provided additional information and one [75] provided additional data.

\subsection{Study Characteristics}

Study characteristics of the 42 trials included in the analyses are presented in Table 1 . The trials used treatment durations from 2 weeks to 18 months, with doses of EPA from 0.013 to $4.050 \mathrm{~g} /$ day, and doses of DHA from 0.010 to $2.700 \mathrm{~g} /$ day. Most trials used marine oil from whole fish, but some used cod liver oil, mussel extracts, seal oil, and krill oil. Thirty-two trials examined RA, 6 trials examined $\mathrm{OA}$, and 4 trials examined other/mixed types of arthritis (juvenile arthritis, number of trials: $k=1$; polyarticular psoriatic arthritis, $k=1$; mixed diagnoses of $\mathrm{RA}$ and/or OA, $k=1$; mixed diagnoses of RA or psoriatic arthritis, $k=1$ ). The trials included 2751 patients with a mean age of 53.8 years (range of mean age of 10-68 years); mean disease duration was 9.7 years (range 2.3-19.0 years). Of the 30 trials with complete data on pain, 25 trials included a patient-reported outcome, and the remaining trials reported tender joint count $(k=2)$, Ritchie articular index $(k=1)$, and NSAID consumption $(k=2)$ (Table S2). 


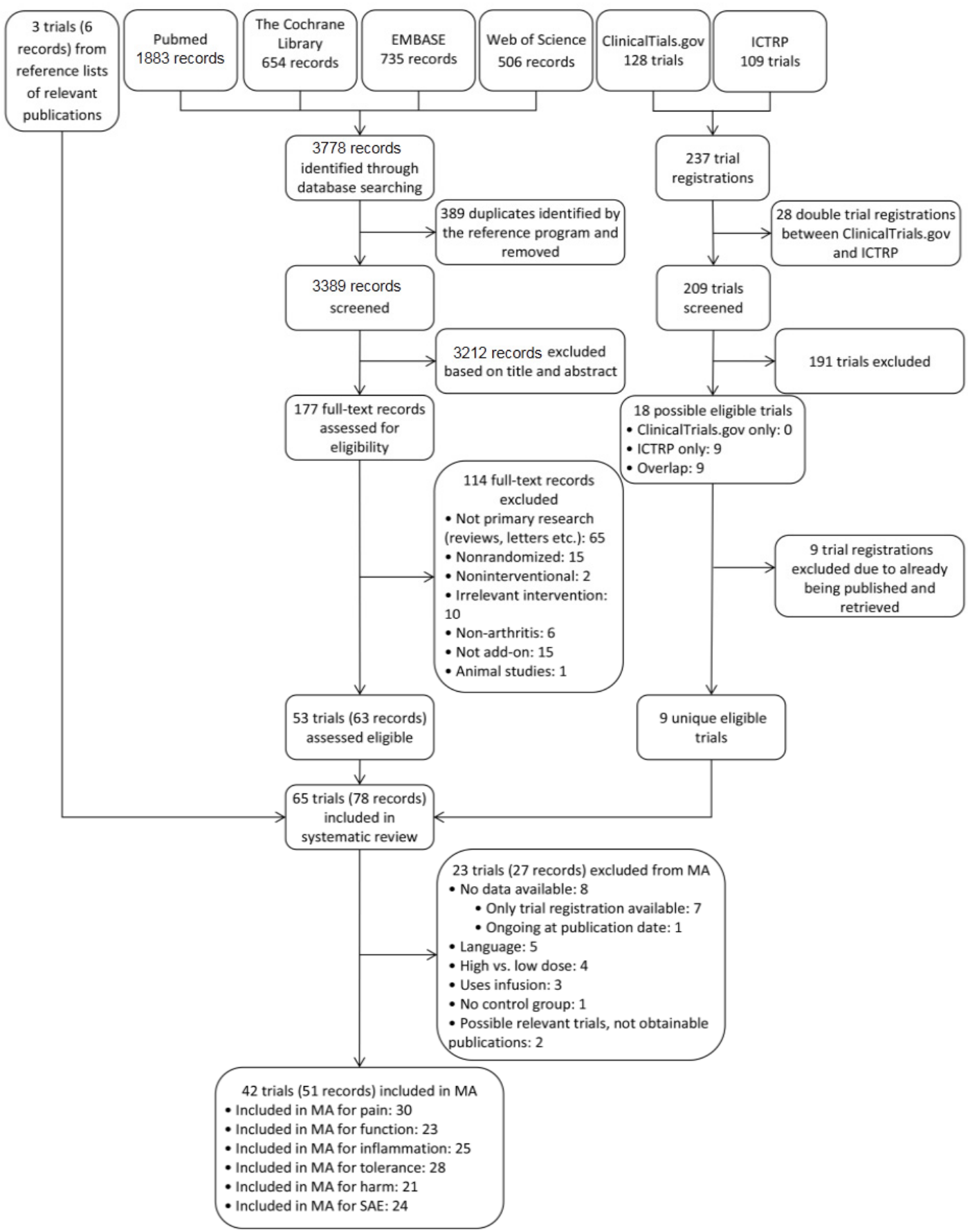

Figure 1. Flow diagram showing the selection of trials. MA: meta-analysis, ICTRP: World Health Organization International Clinical Trial Registry Platform portal, SAE: serious adverse event. 
Table 1. Characteristics of the trials included in the meta-analyses trials presenting complete outcome data on pain are presented in the upper part of the panel.

\begin{tabular}{|c|c|c|c|c|c|c|c|c|c|c|c|c|}
\hline $\begin{array}{c}\text { Author, Year } \\
\text { [Reference Number], } \\
\text { (Trial Registry Number) }\end{array}$ & $\mathrm{N}$ Total & Study Design & $\begin{array}{l}\text { Duration of } \\
\text { Intervention } \\
\text { (Weeks) }\end{array}$ & Diagnosis & $\begin{array}{c}\text { Mean } \\
\text { Age } \\
\text { (Years) }\end{array}$ & $\begin{array}{c}\% \\
\text { Females }\end{array}$ & $\begin{array}{l}\text { Mean Disease } \\
\text { Duration } \\
\text { (Years) }\end{array}$ & $\begin{array}{l}\text { No. of Patients } \\
\text { Receiving } \\
\text { Intervention }\end{array}$ & Intervention & $\begin{array}{l}\text { Dose of EPA + } \\
\text { DHA (g/Day) }\end{array}$ & $\begin{array}{l}\text { No. of Patients } \\
\text { Receiving } \\
\text { Control }\end{array}$ & Control Treatment \\
\hline \multicolumn{13}{|c|}{ Trials with complete data for pain } \\
\hline \multirow[t]{2}{*}{ Belch et al. 1986 [43] } & \multirow[t]{2}{*}{34} & \multirow[t]{2}{*}{ Three-arm, parallel } & \multirow[t]{2}{*}{52} & \multirow[t]{2}{*}{ RA } & \multirow[t]{2}{*}{ na } & \multirow[t]{2}{*}{ na } & \multirow[t]{2}{*}{ na } & $11^{\mathrm{c}}$ & $\begin{array}{c}\mathrm{MO} \text { (whole fish), and EPO } \\
\text { (480 mg GLA) }\end{array}$ & $0.24+\mathrm{na}$ & $12^{\mathrm{c}}$ & Oil (unspecified) ${ }^{\mathrm{k}}$ \\
\hline & & & & & & & & & & & $11^{\mathrm{c}}$ & EPO (540 mg GLA) \\
\hline \multirow{2}{*}{ Belch et al. 1988 [44] } & \multirow{2}{*}{49} & \multirow{2}{*}{ Three-arm, parallel } & \multirow{2}{*}{52} & \multirow{2}{*}{ RA } & \multirow{2}{*}{49.0} & \multirow{2}{*}{29.3} & \multirow{2}{*}{5.0} & 15 & $\begin{array}{c}\mathrm{MO} \text { (whole fish), and EPO } \\
\text { (450 mg GLA) }\end{array}$ & $0.24+\mathrm{na}$ & 18 & Paraffin ${ }^{k}$ \\
\hline & & & & & & & & & & & 16 & EPO (540 mg GLA) \\
\hline Cleland et al. 1988 [45] & 60 & Two-arm, parallel & 12 & RA & 50.5 & 53.3 & 8.3 & 30 & $\mathrm{MO}$ (whole fish) & $3.20+2.00$ & 30 & Olive oil \\
\hline Stammers et al. 1989 [46] & 26 & Two-arm, parallel & 26 & $\mathrm{OA}$ & $52-84$ & 80.8 & na & na & $\mathrm{MO}$ (unspecified) & na & na & Oil (unspecified) \\
\hline \multirow{2}{*}{ Kremer et al. $1990[47]$} & \multirow{2}{*}{64} & \multirow{2}{*}{ Three-arm, parallel $^{c}$} & \multirow{2}{*}{24} & \multirow{2}{*}{ RA } & \multirow{2}{*}{58.3} & \multirow{2}{*}{51.6} & \multirow{2}{*}{13.8} & 19 & $\mathrm{MO}$ (whole fish), high dose & $4.05^{\mathrm{e}}+2.70^{\mathrm{e}}$ & 23 & Olive oil ${ }^{\mathrm{j}}$ \\
\hline & & & & & & & & 22 & $\mathrm{MO}$ (whole fish), low dose & $2.03^{\mathrm{e}}+1.35^{\mathrm{e}}$ & & \\
\hline Tulleken et al. 1990 [48] & 28 & Two-arm, parallel & 13 & RA & 55.0 & 85.7 & 18.0 & 14 & MO (whole fish) & $2.04+1.32$ & 14 & $\begin{array}{c}\text { Coconut oil } \\
\text { w/fish flavor }\end{array}$ \\
\hline $\begin{array}{c}\text { van der Tempel et al. } \\
1990[49,50]\end{array}$ & 16 & Cross-over, no WO & $12^{\mathrm{a}}$ & RA & 53.0 & 56.3 & 12.0 & 16 & MO (whole fish) & $2.04+1.32$ & 16 & $\begin{array}{c}\text { Coconut oil } \\
\text { w/fish aroma }\end{array}$ \\
\hline Espersen et al. 1992 [51] & 32 & Two-arm parallel & 12 & RA & na & na & na & $18^{\mathrm{c}}$ & MO (whole fish) & $2.00+1.20$ & $14^{\mathrm{c}}$ & $\begin{array}{l}\text { Mix (38\% MUFA } \\
\text { and } 21 \% \text { PUFA) }\end{array}$ \\
\hline \multirow{2}{*}{$\begin{array}{l}\text { Kjeldsen-Kragh et al. } \\
1992[52,53]\end{array}$} & \multirow[b]{2}{*}{79} & \multirow{2}{*}{ Three-arm, parallel } & \multirow[b]{2}{*}{16} & & & & & 26 & $\begin{array}{l}\text { MO (whole fish), and } \\
\text { declining NSAID }\end{array}$ & $3.78+1.96$ & 28 & $\begin{array}{c}\text { Corn oil and } \\
\text { declining NSAID } \\
\end{array}$ \\
\hline & & & & RA & 57.3 & 21.5 & 8.5 & 25 & $\begin{array}{l}\text { MO (whole fish), } \\
\text { and NSAID }{ }^{k}\end{array}$ & $3.78+1.96$ & & \\
\hline Magarò et al. 1992 [54] & 20 & Two-arm, parallel & 6 & RA & $25-45$ & 100.0 & na & 10 & $\mathrm{MO}$ (whole fish) & $1.60+1.10$ & 10 & None \\
\hline $\begin{array}{l}\text { Nielsen et al. } \\
1992[55-57]\end{array}$ & 57 & Two-arm, parallel & 12 & RA & $61.0^{\mathrm{b}}$ & na & $5.0^{\mathrm{b}}$ & 29 & $\mathrm{MO}$ (whole fish) & $2.00+1.20$ & 28 & Mix of FA \\
\hline Sköldstam et al. 1992 [58] & 46 & Two-arm, parallel & 26 & RA & 57.0 & 73.9 & 18.0 & 23 & MO (whole fish) & $1.80+1.20$ & 23 & $\begin{array}{l}\text { Maize oil, olive oil } \\
\text { and peppermint oil }\end{array}$ \\
\hline Stammers et al. 1992 [59] & 86 & Two-arm, parallel & 24 & $\mathrm{OA}$ & 68.0 & 72.1 & 15.5 & 44 & MO (cod liver oil) & $0.79+0.70 \mathrm{~g}$ & 42 & Olive oil \\
\hline Geusens et al. 1994 [60] & 90 & Three-arm, parallel & 52 & RA & 57.3 & 52.2 & 10.1 & 30 & $\mathrm{MO}$ (whole fish), high dose & $1.68+0.36$ & 30 & Olive oil ${ }^{j}$ \\
\hline & & & & & & & & 30 & $\mathrm{MO}$ (whole fish), low dose & $0.84+0.18$ & & \\
\hline Kremer et al. 1995 [61] & 66 & Four-arm, parallel & 26 or 30 & RA & 57.5 & 40.9 & 10.5 & $33^{\mathrm{c}}$ & $\begin{array}{l}\mathrm{MO} \text { (whole fish), diclofenac, } \\
\text { and diclofenac placebo }\end{array}$ & $4.29^{e}+2.34^{e}$ & $33^{\mathrm{c}}$ & $\begin{array}{l}\text { Corn oil, diclofenac, } \\
\text { and diclofenac placebo }\end{array}$ \\
\hline Vargová et al. 1998 [62] & 23 & Two-arm, parallel & 21 & JCA & 10.1 & na & na & 13 & $\begin{array}{l}\text { "diet with increased content } \\
\text { of omega-3 PUFA" }\end{array}$ & na & 10 & Unspecified \\
\hline Volker et al. 2000 [63] & 50 & Two-arm, parallel & 15 & RA & 57.0 & $\mathrm{n}$ & 13.5 & 25 & MO (whole fish) & $0.90^{e, g}+0.63^{e, g}$ & 25 & $\begin{array}{l}50: 50 \text { corn oil } \\
\text { and olive oil }\end{array}$ \\
\hline Adam et al. 2003 [64] & 68 & $\begin{array}{c}\text { Two parallel } \\
\text { Tws }\end{array}$ & $13^{\mathrm{a}}$ & RA & 56.8 & 41.2 & 9.6 & 34 & $\mathrm{MO}$ (whole fish), and WD & $0.80^{\mathrm{e}}+0.60^{\mathrm{e}}$ & 34 & Corn oil, and WD \\
\hline & & 8.7 weeks WO & & & 58.0 & 41.2 & 9.5 & 34 & $\mathrm{MO}$ (whole fish), and AID & $0.84^{e}+0.63^{e}$ & 34 & Corn oil, and AID \\
\hline Lau et al. 2004 [65] & 80 & Two-arm, parallel & 24 & Knee OA & 62.5 & 86.3 & 8.9 & 40 & $\mathrm{MO}$ (GLM extract) & na & 40 & Olive oil \\
\hline
\end{tabular}


Table 1. Cont

\begin{tabular}{|c|c|c|c|c|c|c|c|c|c|c|c|c|}
\hline $\begin{array}{c}\text { Author, Year } \\
\text { [Reference Number], } \\
\text { (Trial Registry Number) }\end{array}$ & $\mathrm{N}$ Total & Study Design & $\begin{array}{l}\text { Duration of } \\
\text { Intervention } \\
\text { (Weeks) } \\
\end{array}$ & Diagnosis & $\begin{array}{l}\text { Mean } \\
\text { Age } \\
\text { (Years) }\end{array}$ & $\begin{array}{c}\% \\
\text { Females }\end{array}$ & $\begin{array}{c}\text { Mean Disease } \\
\text { Duration } \\
\text { (Years) }\end{array}$ & $\begin{array}{l}\text { No. of Patients } \\
\text { Receiving } \\
\text { Intervention }\end{array}$ & Intervention & $\begin{array}{l}\text { Dose of EPA + } \\
\text { DHA (g/Day) }\end{array}$ & $\begin{array}{l}\text { No. of Patients } \\
\text { Receiving } \\
\text { Control }\end{array}$ & Control Treatment \\
\hline \multicolumn{13}{|c|}{ Trials with complete data for pain } \\
\hline \multirow{2}{*}{$\begin{array}{l}\text { Sundrarjun et al. } \\
2004 \text { [66] }\end{array}$} & \multirow[b]{2}{*}{60} & \multirow[b]{2}{*}{ Three-arm, parallel } & \multirow[b]{2}{*}{12} & \multirow[b]{2}{*}{ RA } & \multirow[b]{2}{*}{46.9} & \multirow[b]{2}{*}{85.0} & \multirow[b]{2}{*}{4.4} & 23 & MO (whole fish) & $1.88+1.48$ & 23 & Unspecified \\
\hline & & & & & & & & & & na & 14 & None ${ }^{\mathrm{k}}$ \\
\hline \multirow[b]{2}{*}{ Berbert et al. 2005 [67] } & \multirow[b]{2}{*}{55} & \multirow[b]{2}{*}{ Three-arm, parallel } & \multirow[b]{2}{*}{24} & \multirow[b]{2}{*}{ RA } & \multirow[b]{2}{*}{49.0} & \multirow[b]{2}{*}{61.8} & \multirow[b]{2}{*}{15.3} & 18 & MO (whole fish) & $1.80+1.20$ & 17 & Soy oil \\
\hline & & & & & & & & 20 & $\begin{array}{l}\mathrm{MO} \text { (whole fish), } \\
\text { and olive oil }{ }^{\mathrm{k}}\end{array}$ & $1.80+1.20$ & & \\
\hline Madland et al. 2006 [68] & 43 & Two-arm, parallel & 2 & p.a. PsA & 55.0 & 51.2 & $13.0^{\mathrm{b}}$ & 22 & $\mathrm{MO}$ (seal oil) & $2.40+2.60$ & 21 & Soy oil (bottle) \\
\hline Deutsch 2007 [69] & 90 & Two-arm, parallel & 4 & $\mathrm{RA} / \mathrm{CVD} / \mathrm{OA}$ & 55.0 & 47.8 & na & 45 & MO (krill oil) & $0.05+0.03$ & 45 & $\begin{array}{l}\text { Microcrystalline } \\
\text { cellulose }\end{array}$ \\
\hline Galarraga et al. 2008 [70] & 97 & Two-arm, parallel & 36 & RA & 59.5 & 71.1 & 13.0 & 49 & $\begin{array}{l}\text { MO (mix of oil from cod } \\
\text { liver and whole fish) }\end{array}$ & $1.50+0.70$ & 48 & Air filled capsule \\
\hline $\begin{array}{l}\text { Das Gupta et al. } \\
2009 \text { [71] }\end{array}$ & 100 & Two-arm, parallel & 12 & RA & 47.3 & na & na & 50 & $\begin{array}{l}\mathrm{MO} \text { (whole fish), and } \\
\text { indomethacin }\end{array}$ & $1.80^{g}+1.20 \mathrm{~g}$ & 50 & Indomethacin \\
\hline $\begin{array}{l}\text { Gruenwald et al. } \\
22009 \text { [72] } \\
\text { (EUCTR200500366918DE) }\end{array}$ & 177 & Two-arm, parallel & 26 & Knee/hip OA & 62.3 & 63.8 & na & 90 & $\begin{array}{l}\mathrm{MO} \text { (mix of oil from cod } \\
\text { liver and whole fish), and } \\
\text { glucosamine sulfate }\end{array}$ & $0.60^{\mathrm{i}}$ & 87 & $\begin{array}{l}\text { Mix of oils, and } \\
\text { glucosamine sulfate }\end{array}$ \\
\hline $\begin{array}{l}\text { Park et al. 2013 [73] } \\
\text { (NCT01618019) }\end{array}$ & 109 & Two-arm, parallel & 16 & RA & 48.4 & 34.4 & 8.4 & 55 & MO (whole fish) & $2.09+1.17$ & 54 & $\begin{array}{l}\text { Sunflower oil } \\
\text { w/oleic acid }\end{array}$ \\
\hline \multirow[t]{2}{*}{ Araújo et al. 2014 [74] } & \multirow[t]{2}{*}{37} & \multirow[t]{2}{*}{ Three-arm, parallel } & \multirow[t]{2}{*}{26} & \multirow[t]{2}{*}{ RA } & \multirow[t]{2}{*}{ na } & \multirow[t]{2}{*}{ na } & \multirow[t]{2}{*}{ na } & $11^{\mathrm{c}}$ & $\mathrm{MO}$ (unspecified) & na & $15^{\mathrm{c}}$ & na \\
\hline & & & & & & & & 8 & Mediterranean diet ${ }^{\mathrm{k}}$ & na & & \\
\hline Stebbings et al. 2014 [75] & 80 & Two-arm, parallel & 12 & $\begin{array}{l}\text { Knee or } \\
\text { hip OA }\end{array}$ & 66.4 & 55.0 & na & 39 & MO (GLM) & $0.01+0.01$ & 41 & Corn oil \\
\hline $\begin{array}{l}\text { Fu et al. 2015 [76] } \\
\text { (NCT02173587) }\end{array}$ & 50 & Two-arm, parallel & 26 & RA & 57.5 & 60.0 & 7.6 & 25 & $\begin{array}{c}\text { 50:50 MO (HMLE) } \\
\text { and corn oil }\end{array}$ & $0.07^{f}+0.10^{f}$ & 25 & Corn oil \\
\hline \multicolumn{13}{|c|}{ Trials with incomplete data or no data for pain } \\
\hline Kremer et al. 1986 [77] & 36 & $\begin{array}{c}\text { Cross-over, } \\
4 \text { weeks WO }\end{array}$ & $14^{\mathrm{a}}$ & RA & na & na & na & 36 & MO (whole fish) & $2.70+$ na & 36 & Unspecified \\
\hline $\begin{array}{c}\text { Darlington \& Ramsey } \\
1987[40]\end{array}$ & 35 & Two-arm, parallel & 12 & RA & na & na & na & $(17)^{\mathrm{d}}$ & MO (whole fish) & $3.24+2.16$ & $(18)^{d}$ & Olive oil \\
\hline $\begin{array}{c}\text { Hernández-Cruz et al. } \\
1988[78]\end{array}$ & 90 & Two-arm, parallel & 52 & RA & 43.2 & 89.0 & 3.4 & 45 & MO (whole fish) & $1.50+$ na & 45 & Sunflower oil \\
\hline \multirow{2}{*}{ Kremer et al. 1988 [79] } & \multirow{2}{*}{55} & \multirow{2}{*}{ Three-arm, parallel } & 24 & RA & na & na & na & $(18)^{d}$ & MO (whole fish), high dose & $4.05^{\mathrm{e}}+2.70^{\mathrm{e}}$ & $(19)^{d}$ & Olive oil ${ }^{j}$ \\
\hline & & & & & & & & $(18)^{\mathrm{d}}$ & MO (whole fish), low dose & $2.03^{e}+1.35^{e}$ & & \\
\hline Kremer et al. $1993[80]$ & 50 & Two-arm, parallel & 26 or 30 & RA & na & na & na & $(25)^{d}$ & $\begin{array}{l}\mathrm{MO} \text { (whole fish), diclofenac, } \\
\text { and diclofenac placebo }\end{array}$ & $9.75^{e, h}$ & $(25)^{d}$ & $\begin{array}{r}\text { Corn oil, diclofenac, } \\
\text { and diclofenac placebo }\end{array}$ \\
\hline Lau et al. $1993[81,82]$ & 64 & Two-arm, parallel & 52 & RA & 51.4 & 70.3 & 4.2 & 32 & $\mathrm{MO}$ (whole fish) & $1.71+1.14$ & 32 & Air-filled capsule \\
\hline Lau et al. 1995 [83] & 45 & Two-arm, parallel & 26 & RA & $51.0^{\mathrm{b}}$ & 71.1 & $2.3^{\mathrm{b}}$ & 25 & $\mathrm{MO}$ (whole fish) & $1.70+1.10$ & 20 & Air-filled capsule \\
\hline Kolahi et al. 2010 [84] & 90 & Three-arm, parallel & na & RA & na & $\mathrm{Na}$ & na & $(30)^{\mathrm{d}}$ & $\begin{array}{l}\mathrm{MO} \text { (whole fish), and } \\
\text { vitamin E-placebo }\end{array}$ & na & $(30)^{\mathrm{d}}$ & $\begin{array}{l}\text { MO placebo } \\
\text { (unspecified) and } \\
\text { vitamin E placebo }\end{array}$ \\
\hline & & & & & & & & $(30)^{\mathrm{d}}$ & $\begin{array}{l}\mathrm{MO} \text { (whole fish), } \\
\text { and vitamin } \mathrm{E}^{\mathrm{k}}\end{array}$ & & & \\
\hline
\end{tabular}


Table 1. Cont.

\begin{tabular}{|c|c|c|c|c|c|c|c|c|c|c|c|c|}
\hline $\begin{array}{c}\text { Author, Year } \\
\text { [Reference Number, } \\
\text { (Trial Registry Number) }\end{array}$ & $\mathrm{N}$ Total & Study Design & $\begin{array}{l}\text { Duration of } \\
\text { Intervention } \\
\text { (Weeks) }\end{array}$ & Diagnosis & $\begin{array}{c}\text { Mean } \\
\text { Age } \\
\text { (Years) }\end{array}$ & $\begin{array}{c}\% \\
\text { Females }\end{array}$ & $\begin{array}{l}\text { Mean Disease } \\
\text { Duration } \\
\text { (Years) }\end{array}$ & $\begin{array}{l}\text { No. of Patients } \\
\text { Receiving } \\
\text { Intervention }\end{array}$ & Intervention & $\begin{array}{l}\text { Dose of EPA + } \\
\text { DHA (g/Day) }\end{array}$ & $\begin{array}{l}\text { No. of Patients } \\
\text { Receiving } \\
\text { Control }\end{array}$ & Control Treatment \\
\hline \multicolumn{13}{|c|}{ Trials with incomplete data or no data for pain } \\
\hline $\begin{array}{l}\text { Kolahi et al. } 2010 \text { [85-88] } \\
\text { (IRCT138902073812N1) }\end{array}$ & 90 & Two-arm, parallel & 13 & RA & $50.0^{\mathrm{b}}$ & 92.2 & $4.6^{\mathrm{b}}$ & 45 & $\mathrm{MO}$ (whole fish) & $0.18+0.12$ & 45 & Paraffin \\
\hline \multirow{2}{*}{$\begin{array}{c}\text { Dawczynski et al. } \\
2011 \text { [89] (NCT01179971) }\end{array}$} & \multirow{2}{*}{60} & \multirow{2}{*}{ Four-arm, parallel } & \multirow[b]{2}{*}{12} & \multirow{2}{*}{ RA (or PsA) } & \multirow[b]{2}{*}{56.2} & \multirow[b]{2}{*}{71.7} & \multirow[b]{2}{*}{ na } & 15 & $\mathrm{MO}$ (unspecified) & $3.00^{1}$ & 15 & Olive oil \\
\hline & & & & & & & & 15 & $\begin{array}{l}\text { MO (unspecified), } \\
\text { and GLA (1800 mg) }\end{array}$ & $1.58^{1}$ & 15 & GLA (3150 mg) \\
\hline \multirow[t]{2}{*}{$\begin{array}{l}\text { Reed et al. } 2014[90,91] \\
\text { (NCT00072982) }\end{array}$} & \multirow[t]{2}{*}{150} & \multirow[t]{2}{*}{ Three-arm, parallel } & \multirow[t]{2}{*}{78} & \multirow[t]{2}{*}{ RA } & \multirow[t]{2}{*}{59.2} & \multirow[t]{2}{*}{81.3} & \multirow[t]{2}{*}{$8.6^{\mathrm{b}}$} & 53 & $\begin{array}{l}\mathrm{MO} \text { (whole fish), } \\
\text { and borage seed oil }\end{array}$ & $2.10+1.40$ & 52 & $\begin{array}{l}\text { Borage seed oil, and } \\
\text { sunflower seed oil }\end{array}$ \\
\hline & & & & & & & & 45 & $\begin{array}{l}\mathrm{MO} \text { (whole fish), and } \\
\text { sunflower seed oil k }\end{array}$ & $2.10+1.40$ & & \\
\hline \multirow{3}{*}{$\begin{array}{c}\text { Yazdanpanah et al. } \\
2014[92] \\
\text { (IRCT2012102610799N2) }\end{array}$} & \multirow{3}{*}{114} & \multirow{3}{*}{ Six-arm, parallel } & \multirow{3}{*}{6} & \multirow{3}{*}{ Knee OA } & \multirow{3}{*}{ na } & \multirow{3}{*}{ na } & \multirow{3}{*}{ na } & 19 & $\begin{array}{l}\text { Omega-3, } \\
\text { and acetaminophen } \\
\text { and naproxen }\end{array}$ & $1.00^{\mathrm{h}}$ & 19 & $\begin{array}{l}\text { Acetaminophen } \\
\text { and naproxen }\end{array}$ \\
\hline & & & & & & & & 19 & $\begin{array}{c}\text { Omega-3 } \\
\text { and acetaminophen }\end{array}$ & $1.00^{\mathrm{h}}$ & 19 & Acetaminophen \\
\hline & & & & & & & & 19 & Omega- 3 and naproxen & $1.00^{\mathrm{h}}$ & 19 & Naproxen \\
\hline
\end{tabular}

AID: anti-inflammatory diet; CVD: cardiovascular disease; DHA: docosahexaenoic acid; EPA: eicosapentaenoic acid; EPO: evening primrose oil; FA: fatty acid; GLA: gamma-linolenic acid; GLM: green-lipped mussel; HMLE: hard-shelled mussel lipid extract; JCA: juvenile chronic arthritis; MO: marine oil; MUFA: monounsaturated fatty acid; N: number; na: not available; NSAID: nonsteroidal anti-inflammatory drugs, OA: osteoarthritis; p.a. PSA: polyarticular psoriatic arthritis; PUFA: polyunsaturated fatty acid; RA: rheumatoid arthritis; WD: western diet; WO: wash out; Note that the use of the term MO refers to all oils of marine origin (e.g., oil from whole fish, seal, or mussel); ${ }^{\text {a }}$ Duration of first period of cross-over study; ${ }^{b}$ Median; ${ }^{c}$ The numbers of participants receiving the interventions are not clearly stated in the publication, but other information suggests the numbers reported; ${ }^{\mathrm{d}}$ No group sample sizes reported. Assumed equal distribution among groups; ${ }^{\mathrm{e}}$ Based on mean body weight of participants or, if not available, on $75 \mathrm{~kg}$; ${ }^{\mathrm{f}}$ Mean dose given over 26 weeks; ${ }^{\mathrm{g}}$ Estimated from information about EPA/DHA ratio or content in similar products; ${ }^{\mathrm{h}}$ Dose of omega- $3{ }^{i}$ Dose of fish oil; ${ }^{j}$ Placebo group used twice in meta-analysis for two comparisons. Number of patients in the control group divided by two in the analysis; ${ }^{k}$ Group excluded from the meta-analysis. 


\subsection{Risk of Bias in Included Studies}

No trials were judged as having low risk of bias in all nine domains (see Figure 2, bias assessment table, and outcome matrix in Table S3 and S4). Thirteen trials were judged as having high risk of pain outcome-reporting bias, 15 trials were judged to be at high risk of function outcome-reporting bias, and 18 trials were judged to be at high risk of inflammation outcome-reporting bias.

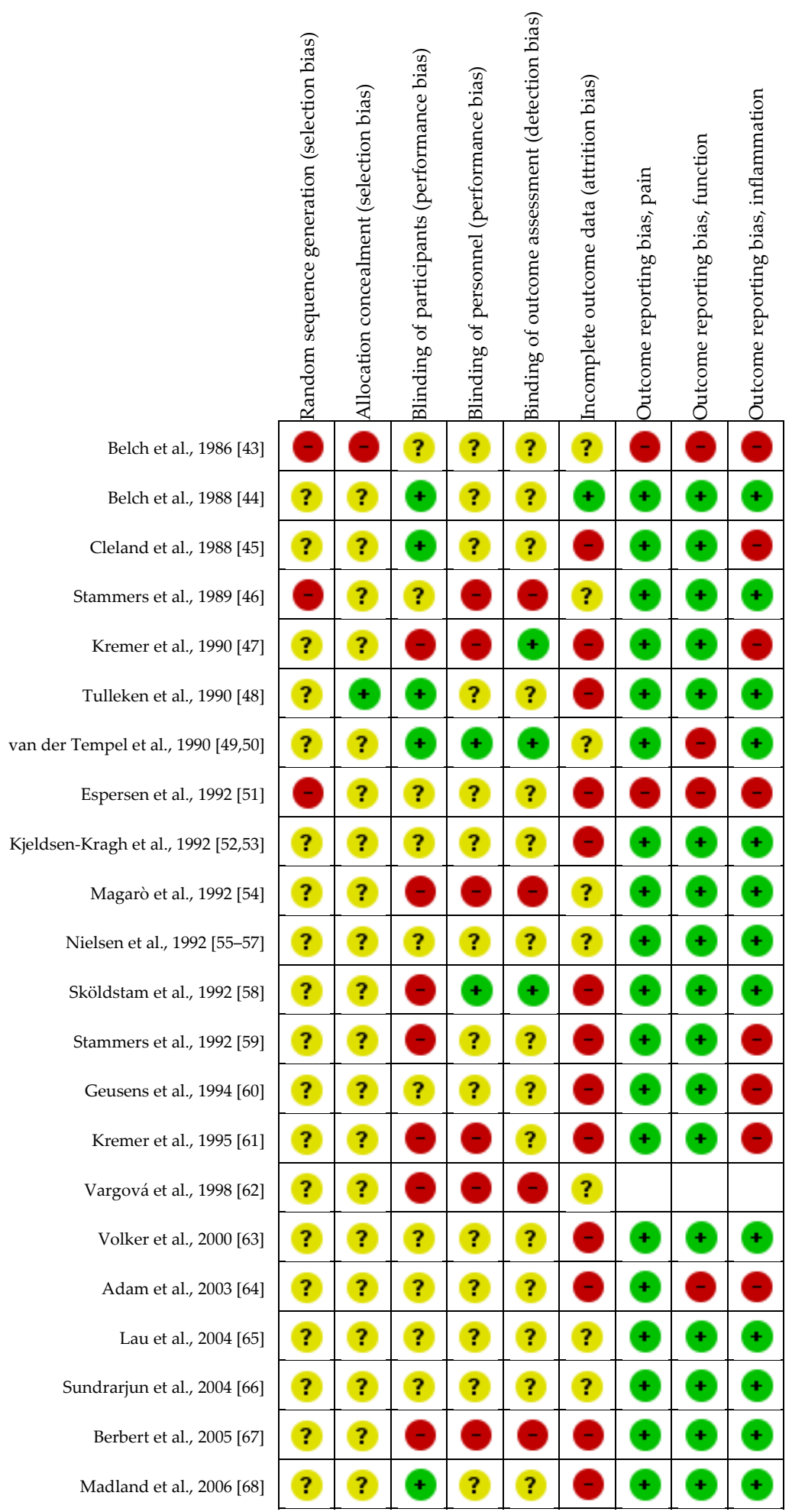

Figure 2. Cont. 


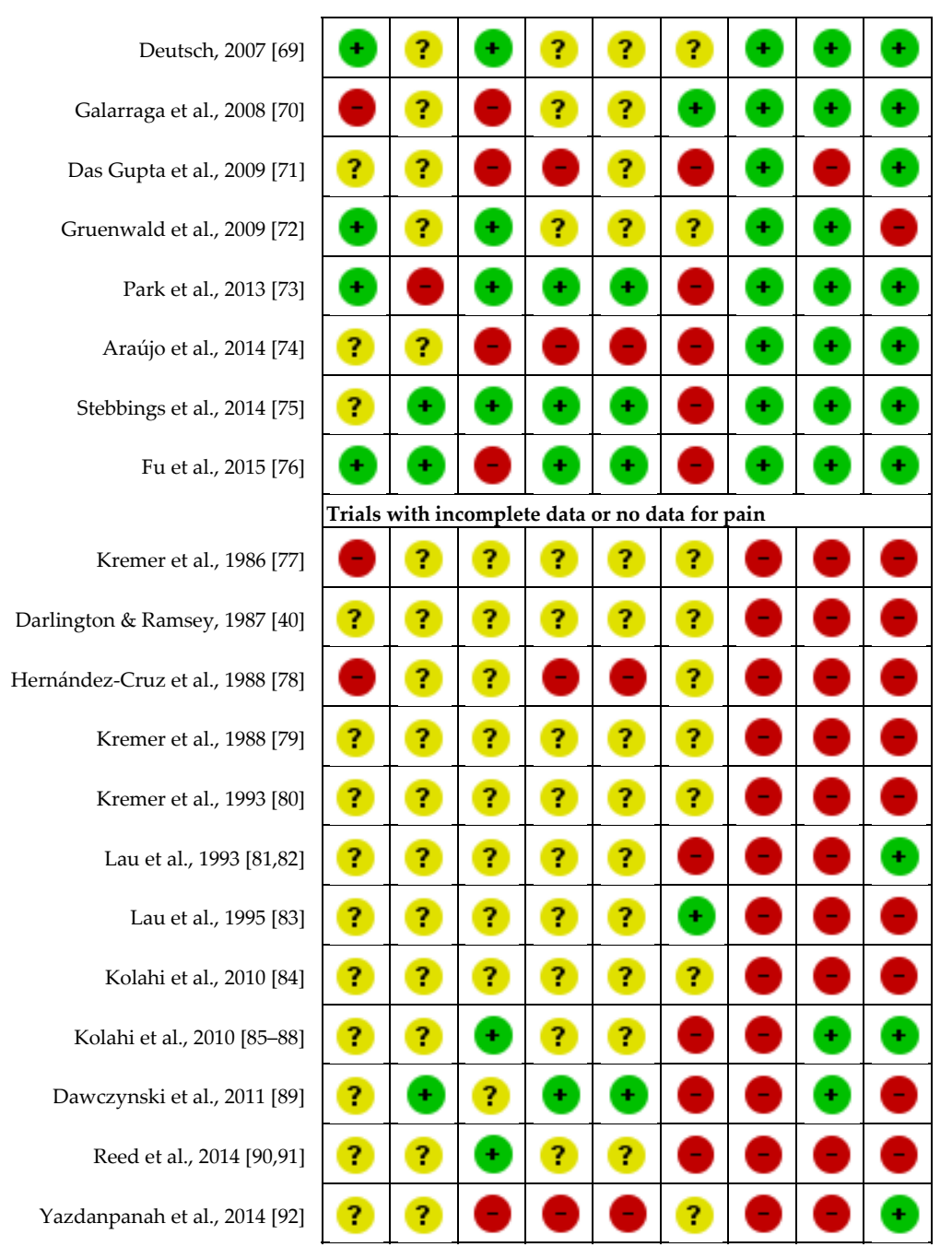

Figure 2. Risk of bias summary figure. $\bullet_{\text {: adequate methodology, }} \boldsymbol{?}$ : unclear methodology, - : inadequate methodology. Risk of outcome reporting bias was not assessed for Vargová et al. (1998) [49], since only the abstract was available in English, and the rest of the article was not in English. Therefore, it was not considered appropriate to assess outcome reporting bias based only on the abstract of a full article.

\subsection{Primary Outcome: Pain}

Thirty trials presented complete data and were included in the meta-analysis on pain. The overall effect estimate corresponded to an SMD of -0.24 ( $95 \%$ confidence interval, CI, -0.42 to -0.07 , $p=0.007$ ), thus indicating a statistically significant pain reducing effect of marine oil (Figure 3 ). This result translates into an improvement of $8 \%$ on a VAS scale. However, not all studies suggested a beneficial effect of marine oil; hence, the effects were highly heterogeneous across studies $\left(I^{2}=63 \%\right)$ with a wide prediction interval for the overall effect estimate (-1.05-0.57). 
Study

Belch et al., 1986 [43]

Belch et al., 1988 [44]

Cleland et al., 1988 [45]

Stammers et al., 1989 [46]

Kremer et al., 1990 [47]

Kremer et al., 1990 [47]

Tulleken et al., 1990 [48]

van der Tempel et al., $1990[49,50]$

Espersen et al., 1992 [51]

Kjeldsen-Kragh et al., 1992 [52,53]

Magarò et al., 1992 [54]

Nielsen et al., 1992 [55-57]

Sköldstam et al., 1992 [58]

Stammers et al., 1992 [59]

Geusens et al., 1994 [60]

Geusens et al., 1994 [60]

Kremer et al., 1995 [61]

Vargová et al., 1998 [62]

Volker et al., 2000 [63]

Adam et al., 2003 [64]

Adam et al., 2003 [64]

Lau et al., 2004 [65]

Sundrarjun et al., 2004 [66]

Berbert et al., 2005 [67]

Madland et al., 2006 [68]

Deutsch, 2007 [69]

Galarraga et al., 2008 [70]

Das Gupta et al., 2009 [71]

Gruenwald et al., 2009 [72]

Park et al., 2013 [73]

Araújo et al., 2014 [74]

Stebbings et al., 2014 [75]

Fu et al., 2015 [76]
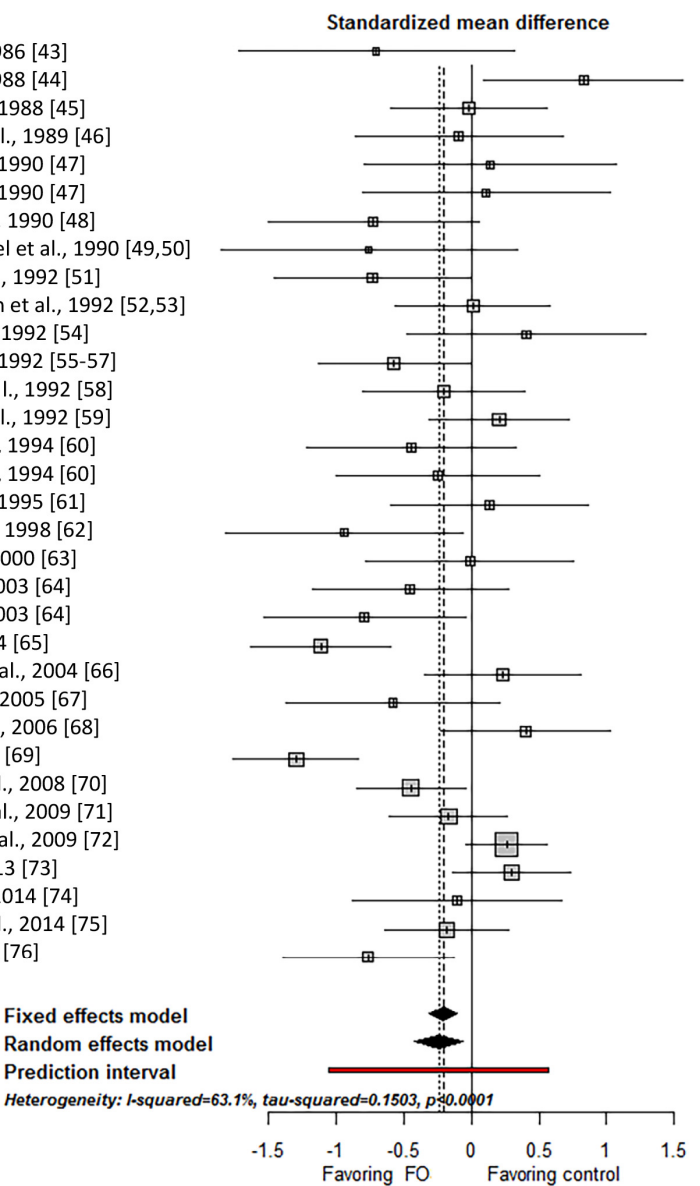

SMD $95 \%-\mathrm{Cl}$ W(fixed) W(random)

$-0.70[-1.72 ; 0.32] \quad 1.0 \% \quad 1.9 \%$

$0.83[0.09 ; 1.57] \quad 2.0 \% \quad 2.8 \%$

$-0.02[-0.60 ; 0.56] \quad 3.2 \% \quad 3.4 \%$

$-0.09[-0.86 ; 0.68] \quad 1.8 \% \quad 2.6 \%$

$0.14[-0.80 ; 1.07] \quad 1.2 \% \quad 2.1 \%$

$0.11[-0.80 ; 1.02] \quad 1.3 \% \quad 2.2 \%$

$-0.72[-1.51 ; 0.06] \quad 1.8 \% \quad 2.6 \%$

$-0.76[-1.85 ; 0.34] \quad 0.9 \% \quad 1.7 \%$

$-0.73[-1.46 ;-0.01] \quad 2.1 \% \quad 2.8 \%$

$0.01[-0.56 ; 0.58] \quad 3.3 \% \quad 3.4 \%$

$0.41[-0.48 ; 1.29] \quad 1.4 \% \quad 2.3 \%$

$-0.57[-1.13 ;-0.01] \quad 3.4 \% \quad 3.5 \%$

$-0.20[-0.80 ; 0.39] \quad 3.0 \% \quad 3.3 \%$

$0.21[-0.31 ; 0.72] \quad 4.0 \% \quad 3.7 \%$

$-0.45[-1.22 ; 0.33] \quad 1.8 \% \quad 2.6 \%$

$-0.25[-1.00 ; 0.51] \quad 1.9 \% \quad 2.7 \%$

$0.14[-0.59 ; 0.87] \quad 2.0 \% \quad 2.8 \%$

$-0.94[-1.82 ;-0.07] \quad 1.4 \% \quad 2.3 \%$

$-0.01[-0.78 ; 0.76] \quad 1.8 \% \quad 2.6 \%$

$-0.45[-1.18 ; 0.27] \quad 2.0 \% \quad 2.8 \%$

$-0.79[-1.54 ;-0.04] \quad 1.9 \% \quad 2.7 \%$

$-1.11[-1.63 ;-0.60] \quad 4.0 \% \quad 3.7 \%$

$0.23[-0.35 ; 0.81] \quad 3.2 \% \quad 3.4 \%$

$-0.58[-1.37 ; 0.21] \quad 1.7 \% \quad 2.6 \%$

$0.40[-0.22 ; 1.03] \quad 2.7 \% \quad 3.2 \%$

$-1.30[-1.76 ;-0.83] \quad 5.0 \% \quad 3.9 \%$

$-0.45[-0.85 ;-0.04] \quad 6.6 \% \quad 4.2 \%$

$-0.17[-0.60 ; 0.27] \quad 5.7 \% \quad 4.0 \%$

$0.26[-0.04 ; 0.56] \quad 12.3 \% \quad 4.6 \%$

$0.29[-0.14 ; 0.73] \quad 5.6 \% \quad 4.0 \%$

$-0.11[-0.88 ; 0.67] \quad 1.8 \% \quad 2.6 \%$

$-0.18[-0.64 ; 0.27] \quad 5.2 \% \quad 3.9 \%$

$-0.76[-1.40 ;-0.13] \quad 2.7 \% \quad 3.1 \%$

$-0.21[-0.31 ;-0.10] \quad 100 \% \quad 100 \%$

$0.24[-0.42 ;-0.07] \quad--\quad 100 \%$

$[-1.05 ; 0.57]$

Figure 3. Forest plot of the effect size for marine oil interventions on pain. Weights are shown for both a random-effects model ("W(random)") and a fixed-effects model ("W(fixed)"). 95\% CI: 95\% confidence interval; MO: marine oil; SMD: standardized mean differences. Note that the use of the term MO refers to all oils of marine origin (e.g., oil from whole fish and mussel oil).

Meta-regression analyses (Table 2), showed that a statistically significant amount of the heterogeneity could be explained by type of diagnosis $(p=0.024)$, supplementation type $(p=0.009)$, dosage of EPA plus DHA $(p=0.016)$, and ratio of EPA/DHA $(p=0.031)$, but not by type of control $(p=0.051)$ or duration $(p=0.074)$. There was a significant effect in patients with RA (22 trials; SMD $-0.21 ; 95 \% \mathrm{CI},-0.42$ to -0.00 ) and other/mixed diagnoses ( 3 trials; SMD $-0.63 ; 95 \% \mathrm{CI}$, -1.20 to -0.06 ), but no effect in patients with OA (5 trials; SMD $-0.17 ; 95 \%$ CI, $-0.57-0.24$ ). All bias domains, except blinding of personnel and blinding of outcome assessors, could also explain a significant amount of the heterogeneity. Post hoc meta-regression analyses for RA separately are provided in Table S5.

A significant positive association was found between SMD and total dose of EPA and DHA (slope $\beta, 0.13$ (g/day) ${ }^{-1}, 95 \% \mathrm{CI}, 0.04-0.22, p=0.006$ ), indicating less effect at higher dose, but there was no duration-response relationship $(p=0.568)$. Plots of the meta-regression analyses are provided in Figure S1.

A post hoc meta-regression analysis was performed exploring differences among the types of marine oil on pain, grouping types of oil as "whole fish" $(k=20)$, "mussel" $(k=3)$, "other" $(k=5$, including cod liver oil, mixture of oil from whole fish and cod liver oil, krill oil, and seal oil), and "unspecified" $(k=3)$. The analysis showed a significant relationship between pain and type of marine oil ( $p=0.012)$, where only the effect of mussel oil was statistically significant on its own, having a beneficial effect. 
Table 2. Results of meta-regression analyses. Analyzed using a random-effects restricted maximum of likelihood (REML)-based meta-regression model.

\begin{tabular}{|c|c|c|c|c|c|c|}
\hline Variable & Total Trials, $k$ & SMD for Pain & $95 \% \mathrm{CI}$ & $\tau^{2}$ & $I^{2}$ & $p$-Value for Interaction \\
\hline All trials & 30 & -0.24 & $(-0.42$ to -0.07$)$ & 0.150 & $63 \%$ & n.a. \\
\hline Diagnoses & & & & 0.147 & $60 \%$ & $0.024^{\mathrm{a}}$ \\
\hline RA & 22 & -0.21 & $(-0.42 \text { to }-0.00)^{a}$ & & & \\
\hline $\mathrm{OA}$ & 5 & -0.16 & $(-0.57$ to 0.24$)$ & & & \\
\hline Other & 3 & -0.63 & $(-1.20 \text { to }-0.06)^{\mathrm{a}}$ & & & \\
\hline Supplementation type & & & & 0.137 & $60 \%$ & $0.009^{\mathrm{a}}$ \\
\hline Capsule & 23 & -0.25 & $(-0.44 \text { to }-0.06)^{a}$ & & & \\
\hline Bottle & 3 & 0.19 & $(-0.37$ to 0.75$)$ & & & \\
\hline Unclear & 4 & -0.61 & $(-1.16 \text { to }-0.05)^{\text {a }}$ & & & \\
\hline Type of control & & & & 0.148 & $60 \%$ & 0.051 \\
\hline PUFA w/o EPA and DHA & 11 & -0.12 & ( -0.41 to 0.17$)$ & & & \\
\hline Non-PUFA oils & 10 & -0.30 & $(-0.59 \text { to }-0.01)^{a}$ & & & \\
\hline Non-oils & 3 & -0.63 & $(-1.13 \text { to }-0.12)^{a}$ & & & \\
\hline Unclear & 3 & -0.01 & $(-0.59$ to 0.56$)$ & & & \\
\hline None & 3 & -0.21 & $(-0.87$ to 0.44$)$ & & & \\
\hline Duration & & & & 0.165 & $63 \%$ & 0.074 \\
\hline$<12$ weeks & 3 & -0.30 & $(-0.89$ to 0.30$)$ & & & \\
\hline$\geq 12$ weeks and $<24$ weeks & 12 & -0.25 & $(-0.53$ to 0.03$)$ & & & \\
\hline$\geq 24$ weeks & 15 & -0.23 & $(-0.48$ to 0.03$)$ & & & \\
\hline Unspecified & 0 & & & & & \\
\hline Ratio of EPA/DHA & & & & 0.153 & $61 \%$ & $0.031^{\mathrm{a}}$ \\
\hline Ratio of EPA/DHA $\leq 1.5$ & 12 & -0.12 & $(-0.40$ to 0.15$)$ & & & \\
\hline Ratio of EPA/DHA of $>1.5$ & 11 & -0.38 & $(-0.67 \text { to }-0.10)^{a}$ & & & \\
\hline Unspecified & 7 & -0.23 & $(-0.62$ to 0.17$)$ & & & \\
\hline Dosage of EPA plus DHA & & & & 0.138 & $58 \%$ & $0.016^{\mathrm{a}}$ \\
\hline$<2.6 \mathrm{~g} /$ day & 8 & -0.45 & $(-0.75 \text { to }-0.15)^{a}$ & & & \\
\hline$\geq 2.6 \mathrm{~g} /$ day and $<3.6 \mathrm{~g} /$ day & 11 & -0.21 & $(-0.51$ to 0.09$)$ & & & \\
\hline$\geq 3.6 \mathrm{~g} /$ day & 4 & 0.13 & $(-0.32$ to 0.57$)$ & & & \\
\hline Unspecified & 7 & -0.22 & $(-0.60$ to 0.16$)$ & & & \\
\hline Source of marine oil & & & & 0.130 & $57 \%$ & $0.011^{\mathrm{a}}$ \\
\hline Whole fish & 19 & -0.17 & $(-0.38$ to 0.05$)$ & & & \\
\hline Mussel & 2 & -0.95 & $(-1.60 \text { to }-0.31)^{a}$ & & & \\
\hline Other & 6 & -0.19 & $(-1.53$ to 0.16$)$ & & & \\
\hline Unspecified & 3 & -0.35 & $(-0.97$ to 0.26$)$ & & & \\
\hline \multicolumn{7}{|l|}{ Bias domains } \\
\hline \multicolumn{4}{|c|}{ Random sequence generation (selection bias) } & 0.160 & $61 \%$ & $0.043^{\mathrm{a}}$ \\
\hline Adequate & 4 & -0.33 & ( -0.78 to 0.13$)$ & & & \\
\hline Unclear & 22 & -0.19 & $(-0.40$ to 0.02$)$ & & & \\
\hline Inadequate & 4 & -0.48 & $(-1.00$ to 0.05$)$ & & & \\
\hline \multicolumn{4}{|c|}{ Allocation concealment (selection bias) } & 0.155 & $61 \%$ & $0.039^{\mathrm{a}}$ \\
\hline Adequate & 3 & -0.52 & $(-1.09$ to 0.05$)$ & & & \\
\hline Unclear & 25 & -0.22 & $(-0.42 \text { to }-0.03)^{a}$ & & & \\
\hline Inadequate & 2 & -0.03 & $(-0.76$ to 0.70$)$ & & & \\
\hline \multicolumn{4}{|c|}{ Blinding of participants (performance bias) } & 0.149 & $61 \%$ & $0.026^{\mathrm{a}}$ \\
\hline Adequate & 9 & -0.10 & $(-0.42$ to 0.22$)$ & & & \\
\hline Unclear & 10 & -0.41 & $(-0.70 \text { to }-0.11)^{a}$ & & & \\
\hline Inadequate & 11 & -0.20 & $(-0.49$ to 0.10$)$ & & & \\
\hline \multicolumn{4}{|c|}{ Blinding of personnel (performance bias) } & 0.163 & $63 \%$ & 0.060 \\
\hline Adequate & 5 & -0.25 & $(-0.70$ to 0.20$)$ & & & \\
\hline Unclear & 17 & -0.29 & $(-0.52 \text { to }-0.05)^{a}$ & & & \\
\hline Inadequate & 8 & -0.13 & $(-0.50$ to 0.24$)$ & & & \\
\hline \multicolumn{4}{|c|}{ Incomplete outcome data (attrition bias) } & 0.152 & $60 \%$ & $0.020^{\mathrm{a}}$ \\
\hline Adequate & 2 & 0.06 & $(-0.61$ to 0.73$)$ & & & \\
\hline Unclear & 10 & -0.44 & $(-0.77 \text { to }-0.12)^{a}$ & & & \\
\hline Inadequate & 18 & -0.18 & $(-0.40$ to 0.04$)$ & & & \\
\hline \multicolumn{4}{|c|}{ Outcome reporting (outcome reporting bias for pain) } & 0.145 & $61 \%$ & $0.016^{\mathrm{a}}$ \\
\hline Adequate & 27 & -0.20 & $(-0.38 \text { to }-0.02)^{a}$ & & & \\
\hline Unclear & 1 & -0.94 & $(-2.10$ to 0.21$)$ & & & \\
\hline Inadequate & 2 & -0.72 & $(-1.52$ to 0.08$)$ & & & \\
\hline Funding source & & & & 0.149 & $61 \%$ & $0.031^{\mathrm{a}}$ \\
\hline Industry only & 8 & -0.10 & $(-0.41$ to 0.21$)$ & & & \\
\hline Mixed & 8 & -0.10 & $(-0.44$ to 0.25$)$ & & & \\
\hline Nonprofit only & 4 & -0.41 & $(-0.87$ to 0.05$)$ & & & \\
\hline Not reported & 7 & -0.31 & $(-0.70$ to 0.08$)$ & & & \\
\hline Unclear & 3 & -0.80 & $(-1.42 \text { to }-0.16)^{a}$ & & & \\
\hline
\end{tabular}

95\% CI: 95\% confidence interval, $I^{2}$ : heterogeneity, SMD: standardized mean difference, $\mathrm{k}$ : number of trials, $\tau^{2}$ : estimated between-study variance; ${ }^{\mathrm{a}} p<0.05$. 


\subsection{Secondary Outcomes: Inflammation and Function}

A total of 23 and 25 trials provided complete data on function and inflammation, respectively. Meta-analysis showed no overall effect of marine oil on function (SMD $-0.01 ; 95 \%$ CI, $-0.19-0.18$; $p=0.953)$ and moderate heterogeneity across studies $\left(I^{2}=60 \%\right)$. Including studies with incomplete data on function and a high risk of outcome-reporting bias, using null-imputations, yielded similar results (SMD $-0.01 ; 95 \% C I,-0.13-0.10 ; p=0.808)$.

The overall effect of marine oil on inflammation was significant (SMD $-0.28 ; 95 \% \mathrm{CI},-0.51-0.06$; $p=0.013)$, corresponding to an effect of $-1.7 \mathrm{mg} / \mathrm{dL}(-3.0$ to $-0.4 \mathrm{mg} / \mathrm{dL}) \mathrm{CRP}$, with substantial heterogeneity across studies $\left(I^{2}=70 \%\right)$. Including studies with incomplete data on inflammation and a high risk of outcome-reporting bias yielded similar results (SMD $-0.16 ; 95 \% \mathrm{CI},-0.29$ to $-0.02 ; p=0.021$ ). Forest plots for function and inflammation are provided in Figures S2 and S3, and meta-regression analyses for function and inflammation are provided in Tables S6 and S7.

\subsection{Tolerance and Safety}

A total of 28, 21, and 24 trials with complete data were included in the analysis of tolerance, withdrawals due to adverse events, and SAEs, respectively. For all three outcomes, there were no differences between the intervention and the control group, with an RR of 1.00 (95\% CI, 0.96-1.03; $p=0.814), 0.82(95 \%$ CI, 0.57-1.17; $p=0.279)$ and $0.75(95 \% \mathrm{CI}, 0.43-1.30 ; p=0.308)$, respectively. Low heterogeneity was present for all three outcomes $\left(I^{2}=6.5 \%, 0 \%\right.$, and $0 \%$, respectively).

\subsection{Assessment of Reporting Bias}

Visual inspection of the funnel plot for pain did not show any signs of asymmetry, and the result of the Egger test was nonsignificant (intercept $\alpha=-0.91 ; 95 \% \mathrm{CI},-2.75-0.93 ; p=0.339$ ). Similar results were found for function (intercept $\alpha=0.83 ; 95 \% \mathrm{CI},-1.25-2.92 ; p=0.442$ ) and inflammation (intercept $\alpha=0.06 ; 95 \% \mathrm{CI},-3.91-1.75, p=0.462)$, but asymmetry was present for tolerance, withdrawals due to adverse events, and SAEs, likely due to outcome-reporting bias. The funnel plots are provided in Figures S4-S9.

Sensitivity analyses using fixed-effect models yielded similar results as the random-effects models for pain, function, and inflammation, indicating limited effect of potential small-study bias. Including trials with incomplete data on pain and a high risk of outcome-reporting bias yielded a slightly lower but still statistically significant effect (42 trials, SMD $-0.16 ; 95 \% \mathrm{CI},-0.28$ to $-0.03 ; p=0.012$; forest plot is provided in Figure S10). The effect size was influenced by type of pain outcome, as shown by a post hoc meta-regression analysis $(p=0.005)$, with the effect size being nonsignificant for trials reporting patient-reported pain (SMD $-0.18 ; 95 \% \mathrm{CI},-0.37-0.00 ; p=0.051$ ) and significant for trials reporting non-patient-reported pain (SMD $-0.65 ; 95 \% \mathrm{CI},-0.65$ to $-0.16 ; p=0.009$ ). However, two of the latter trials were deemed low risk of outcome-reporting bias, and the effect on pain was still significant when excluding trials with both non-patient-reported pain outcome and high/unclear outcome-reporting bias (corresponding to 'Adequate' outcome reporting in Table 2).

The GRADE evidence profile with reasons for downgrading is provided in Table 3. 
Table 3. GRADE evidence profile.

\begin{tabular}{|c|c|c|c|c|c|c|c|c|c|c|}
\hline \multicolumn{6}{|c|}{ Quality Assessment } & \multicolumn{2}{|c|}{ No. of Patients } & \multirow{2}{*}{\multicolumn{2}{|c|}{$\begin{array}{l}\text { Effect } \\
\text { Absolute }(95 \% \mathrm{CI})\end{array}$}} & \multirow{2}{*}{ Quality } \\
\hline No. of Trials & Risk of Bias & Inconsistency & Indirectness & Imprecision & $\begin{array}{c}\text { Other } \\
\text { Considerations }\end{array}$ & MO & Non-MO & & & \\
\hline \multicolumn{11}{|c|}{ Pain } \\
\hline 30 & Serious $(-1)^{\text {a }}$ & Serious $(-1)^{\mathrm{b}}$ & Not serious & Not serious & None & 781 & 728 & - & $\begin{array}{c}\text { SMD } 0.24 \text { lower } \\
(0.42 \text { lower to } 0.07 \text { lower })\end{array}$ & $\oplus \oplus \mathrm{LOW}$ \\
\hline RA 22 & Serious $(-1)^{a}$ & Not serious & Not serious & Not serious & None & 499 & 457 & - & $\begin{array}{c}\text { SMD } 0.21 \text { lower } \\
\text { (0.42 lower to }-0.004 \text { lower) }\end{array}$ & $\oplus \oplus \oplus$ MODERATE \\
\hline OA 5 & Serious $(-1)^{\text {a }}$ & Serious $(-1)^{\mathrm{b}}$ & Not serious & Serious $(-1)^{\mathrm{d}}$ & None & 205 & 198 & - & $\begin{array}{c}\text { SMD } 0.16 \text { lower } \\
\text { (0.57 lower to } 0.24 \text { higher) }\end{array}$ & ๑VERY LOW \\
\hline Other 3 & Serious $(-1)^{a}$ & Serious $(-1)^{b}$ & Serious $(-1)^{\mathrm{c}}$ & Serious $(-1)^{\mathrm{d}}$ & None & 77 & 73 & - & $\begin{array}{c}\text { SMD } 0.63 \text { lower } \\
(1.20 \text { lower to }-0.06 \text { lower })^{\dagger}\end{array}$ & ๑VERY LOW \\
\hline \multicolumn{11}{|c|}{ Function (assessed with functional tests) } \\
\hline 23 & Serious $(-1)^{\mathrm{a}}$ & Not serious & Not serious & Not serious & None & 666 & 611 & - & $\begin{array}{c}\text { SMD } 0.01 \text { lower } \\
\text { (0.19 lower to } 0.18 \text { higher) }\end{array}$ & $\oplus \oplus \oplus$ MODERATE \\
\hline \multicolumn{11}{|c|}{ Inflammation } \\
\hline 25 & Serious $(-1)^{a}$ & Serious $(-1)^{\mathrm{b}}$ & Not serious & Not serious & None & 581 & 573 & - & $\begin{array}{c}\text { SMD } 0.28 \text { lower } \\
\text { (0.51 lower to } 0.06 \text { lower) }\end{array}$ & $\oplus \oplus$ LOW \\
\hline \multicolumn{11}{|c|}{ Tolerance } \\
\hline 28 & Serious $(-1)^{\text {a }}$ & Not serious & Not serious & Not serious & None & 951 & 899 & $\begin{array}{c}\text { RR } 1.00 \\
(0.96 \text { to } 1.03) \\
\end{array}$ & $\begin{array}{l}3 \text { fewer per } 1000 \\
\text { (from } 27 \text { fewer to } 22 \text { more) }\end{array}$ & $\oplus \oplus \oplus$ MODERATE \\
\hline \multicolumn{11}{|c|}{ Number of withdrawals due to adverse events } \\
\hline 21 & Serious $(-1)^{\mathrm{a}}$ & Not serious & Not serious & Not serious & None & 751 & 691 & $\begin{array}{c}\text { RR } 0.82 \\
(0.57 \text { to } 1.17) \\
\end{array}$ & $\begin{array}{c}16 \text { fewer per } 1000 \\
\text { (from } 36 \text { fewer to } 15 \text { more) }\end{array}$ & $\oplus \oplus \oplus$ MODERATE \\
\hline \multicolumn{11}{|c|}{ Serious adverse events } \\
\hline 24 & Serious $(-1)^{\mathrm{a}}$ & Not serious & Not serious & Serious $(-1)^{\mathrm{d}}$ & None & 890 & 839 & $\begin{array}{c}\text { RR } 0.75 \\
\text { (0.43 to } 1.30)\end{array}$ & $\begin{array}{l}\quad 8 \text { fewer per } 1000 \\
\text { (from } 19 \text { fewer to } 10 \text { more) }\end{array}$ & $\oplus \oplus \mathrm{LOW}$ \\
\hline
\end{tabular}

Table made with GRADEpro, (Computer program on www.gradepro.org), version July 8, 2015. McMaster University, 2014; 95\% CI: 95\% confidence interval, MO: marine oil, OA osteoarthritis, RA: rheumatoid arthritis, RR: relative risk, SMD: standardized mean difference. Note that the use of the term MO refers to all oils of marine origin (e.g., oil from whole fish, seal, or mussel); ${ }^{\text {a }}$ The major study limitations were not using intention-to-treat, and unclear random sequence generation, allocation concealment, blinding of personnel, blinding of outcome assessment in most of the trials. None of the trials were rated adequate for all bias domains, with the maximum being five out of seven for pain, function and inflammation. For the "other" group, the maximum was three out of seven. For tolerance, number of withdrawals due to adverse events, and serious adverse events, the maximum was four out of six; ${ }^{\mathrm{b}}$ Unexplained heterogeneity and wide prediction intervals. For pain, a maximum of $13 \%$ of the heterogeneity could be explained by the meta-regression analyses. For inflammation, a maximum of $29 \%$ of the heterogenety coun For ing separate analy investigate the heterogeneity; ${ }^{c}$ Indirect evidence, from including an intervention of "diet with increased content of omega-3 PUFA" [62]; ${ }^{\mathrm{d}}$ Wide $95 \%$ confidence interval, i.e., including both harmful and beneficial effects. Trials including patients with OA and "other" included only 403 and 150 patients, respectively, which is below the calculated optimal information size (OIS) of 786 patients; ${ }^{\mathrm{e}}$ Calculated by 1000 ACR.(1 - RR), where ACR is the assumed control risk, calculated by (number of events in control group)/(size of control group) 


\section{Discussion}

The present systematic review and meta-analysis indicated an effect of marine oil on arthritis, although with a considerable heterogeneity across the studies. Twenty-two trials $(\mathrm{N}=956)$ with complete data on pain assessed the effect of marine oil in patients with RA, for whom a favorable effect was seen, though the confidence in the estimate is considered moderate. Only five trials $(\mathrm{N}=403)$ assessed the effect of marine oil in patients with $\mathrm{OA}$, and only three trials $(\mathrm{N}=150)$ assessed the effect in patients with other arthritis diagnoses; we rated the evidence to be of low grade for both patient groups. Hence, the evidence was not sufficiently robust to determine the effect of marine oil in patients with diagnoses other than RA. The results for the OA and other/mixed diagnosis groups were highly heterogeneous $\left(I^{2}=82 \%\right.$ and $89 \%$, respectively). Osteoarthritis has varying degrees of inflammation [4]; hence, in the future it might be appropriate to distinguish between OA with higher and lower degrees of inflammation.

A significant, beneficial effect on pain was found for marine oil with an EPA/DHA ratio >1.5, suggesting that EPA is more beneficial than DHA, which could be due to a more potent effect mechanism for EPA than for DHA [94]. An inverse dose-response relationship was found, suggesting less pain reduction with higher doses, which is against expectations. Previously, RA symptoms have been reduced at a total EPA and DHA dose of $\geq 2.6 \mathrm{~g} /$ day over $\geq 12$ weeks, and the higher the doses the less time was needed to demonstrate the effect [95]. This was not supported by our findings, as a dose of $\geq 2.6 \mathrm{~g} /$ day was not significant in the meta-regression. However, the results should be interpreted with caution. This finding could be explained by a possible saturation dose-response-relationship, as different background diets provide varying amounts of EPA and DHA. One of the four trials applying the highest doses of EPA and DHA was conducted in a Norwegian population [52], which already has a high intake of fish oil compared to e.g., an American population [52], possibly making it more difficult to detect an effect. Two of the trials applying the highest doses reduced the patients' intake of NSAIDs during the trial $[47,52]$, which also might make it more difficult to detect an effect on pain. In addition, there is uncertainty about the actual doses ingested, since some of the trials included non-compliant patients and some did not measure compliance. The optimal type of marine oil could not be established because only few trials included marine oil from sources other than whole fish. Conclusions regarding dosage, duration, and ratio of EPA/DHA did not change by including only trials with RA patients.

Assessing types of adverse effects was beyond the scope of this systematic review, but no differences were found between intervention and control groups in terms of tolerance, withdrawals due to adverse events, and SAEs, which is in accordance with the findings of a systematic review of fish oil administration in older adults [96].

In general, the trials showed serious study limitations and a large degree of heterogeneity (i.e., "disagreement" with respect to the effect of marine oil). Heterogeneity was expected from pooling different diagnoses, types of marine oils, and measures within each outcome. The pooling, however, did increase the power of the analyses. When exploring the heterogeneity with meta-regression analyses, the majority of the heterogeneity remained unexplained for pain and inflammation, and quality of evidence for these two outcomes was therefore rated as low. When only trials with RA patients were included in a post hoc meta-analysis of pain, the heterogeneity was low $\left(I^{2}=32 \%\right)$, i.e., these trials generally agree on the effect. Post hoc analyses showed that heterogeneity for function was explained to a large degree by diagnosis and/or trial duration, and the quality of evidence was rated as moderate.

Poor reporting was a frequent issue in the trials, limiting their inclusion in the meta-analysis and making their methods nontransparent. Consequently, risk of bias may have been high, although it was frequently judged as unclear. Publication bias and outcome-reporting bias were also potential limitations. However, funnel plots, Egger tests, and sensitivity analyses including trials with incomplete data did not show evidence that these bias types were affecting the estimates for pain, function, or inflammation. 
To our knowledge this is the first comprehensive systematic review and meta-analysis regarding the effect of marine oil on arthritis. In contrast to previous meta-analyses [14-17], we included all types of arthritis, all types of marine oil (i.e., oils of any marine origin), and did not include vegetable sources of n-3 PUFA (e.g., flaxseed oil). In addition, we applied extensive search strategies.

\section{Conclusions}

In conclusion, this meta-analysis suggests a small favorable effect of marine oil in reducing pain in patients with arthritis (SMD < -0.2) [97], but the evidence was of low quality. There is moderate quality evidence for an effect in RA patients. In contrast, the effect was statistically non-significant in patients with OA, but our confidence in the estimate is very low. Thus, more research is needed in order to provide evidence for firm conclusions regarding the effect of marine oil in OA and other types of arthritis, but also regarding the optimal dose, ratio of EPA and DHA, and which type of marine oil is preferable. If RA patients would like to try marine oil (i.e., oil from any marine origin), the results suggest a product with an EPA/DHA ratio $>1.5$, and there do not seem to be adverse effects.

Supplementary Materials: The following are available online at http://www.mdpi.com/2072-6643/9/1/42/s1, Section S1: Reference Lists of Included Trials in the Meta-Analysis, Section S2: Reference Lists of Excluded Trials prior to the Systematic Review, Section S3: Reference Lists of Excluded Trials prior to the Meta-Analysis, Table S1: Search Strategies for all Databases, Table S2: Outcome Matrix, Table S3: Bias Assessment Table, Table S4: Outcome reporting bias Assessment, Table S5: Meta-Regression Analysis on Pain for Rheumatoid Arthritis Patients, Table S6: Meta-Regression Analysis on Function, Table S7: Meta-Regression Analysis on Inflammation, Figure S1: Meta-regression analysis (SMDs plotted against EPA plus DHA and duration of treatment), Figure S2: Forest Plot for Function, Figure S3: Forest Plot for Inflammation, Figure S4: Funnel Plot for Pain, Figure S5: Funnel Plot for Function, Figure S6: Funnel Plot for Inflammation, Figure S7: Funnel Plot for Tolerance, Figure S8: Funnel Plot for Withdrawals due to Adverse Events, Figure S9: Funnel Plot for Serious Adverse Events, Figure S10: Forest Plot for Pain Including Trials with Incomplete Data, Figure S11: Forest Plot for Function Including Trials with Incomplete Data, Figure S12: Forest Plot for Inflammation Including Trials with Incomplete Data.

Acknowledgments: We thank the Parker Institute, Bispebjerg and Frederiksberg Hospital, and The Oak Foundation for creating the possibility and right settings for this research. Additionally we thank Kerry Dwan, Institute of Translational Medicine, University of Liverpool, for clarifying issues in the tool for assessing outcome-reporting bias 27; Guido Schwarzer, Institute of Medical Biometry and Medical Informatics, University Medical Center Freiburg, Germany, for providing support for the statistical program R; Christian Ritz, Department of Nutrition, Exercise and Sports, University of Copenhagen, Denmark, for providing statistical support and support for the statistical program R; and several authors of the included trials, especially Simon Stebbings, Department of Medicine, Dunedin School of Medicine, University of Otago, Dunedin, New Zealand, for providing additional trial information. None of the aforementioned persons received any compensation for their contributions. This research received no specific grant from any funding agency in the public, commercial, or not-for-profit sectors. This research was indirectly funded by The Oak Foundation. M.E. Suarez-Almazor has a Midcareer Investigator Award from the National Institute of Arthritis and Musculoskeletal and Skin Diseases (K24 AR053593). Neither the Oak Foundation nor sponsors had a role in any aspect of the current study, including the design and conduct of the study; collection, management, analysis, and interpretation of the data; and preparation, review, and approval of the manuscript.

Author Contributions: Designed research: Ninna K. Senftleber, Sabrina M. Nielsen, Jens R. Andersen, Henning Bliddal, Simon Tarp, Lotte Lauritzen, Robin Christensen; Conducted research: Ninna K. Senftleber, Sabrina M. Nielsen, Robin Christensen; Analyzed data or performed statistical analysis: Ninna K. Senftleber, Sabrina M. Nielsen; Wrote paper: Ninna K. Senftleber, Sabrina M. Nielsen, Jens R. Andersen, Henning Bliddal, Simon Tarp, Lotte Lauritzen, Daniel E. Furst, Maria E. Suarez-Almazor, Anne Lyddiatt, Robin Christensen; Had primary responsibility for final content: Ninna K. Senftleber, Sabrina M. Nielsen, Robin Christensen.

Conflicts of Interest: The authors declare no conflict of interest.

\section{References}

1. Dieppe, P.A.; Lohmander, L.S. Pathogenesis and management of pain in osteoarthritis. Lancet 2005, 365, 965-973. [CrossRef]

2. McInnes, I.B.; Schett, G. The pathogenesis of rheumatoid arthritis. N. Engl. J. Med. 2011, 365, $2205-2219$. [CrossRef] [PubMed] 
3. Barbour, K.E.; Helmick, C.G.; Theis, K.A.; Murphy, L.B.; Hootman, J.M.; Brady, T.J.; Cheng, Y.J. Prevalence of doctor-diagnosed arthritis and arthritis-attributable activity limitation-united states, 2010-2012. Morb. Mortal. Wkly. Rep. 2013, 62, 869-873.

4. Sokolove, J.; Lepus, C.M. Role of inflammation in the pathogenesis of osteoarthritis: Latest findings and interpretations. Ther. Adv. Musculoskelet. Dis. 2013, 5, 77-94. [CrossRef] [PubMed]

5. Scanzello, C.R.; Goldring, S.R. The role of synovitis in osteoarthritis pathogenesis. Bone 2012, 51, $249-257$. [CrossRef] [PubMed]

6. Walsh, D.A.; McWilliams, D.F. Pain in rheumatoid arthritis. Curr. Pain Headache Rep. 2012, 16, 509-517. [CrossRef] [PubMed]

7. Heiberg, T.; Kvien, T.K. Preferences for improved health examined in 1024 patients with rheumatoid arthritis: Pain has highest priority. Arthritis Rheum. 2002, 47, 391-397. [CrossRef] [PubMed]

8. Desai, R.J.; Agarwal, S.J.; Aparasu, R.R. Drug use trends for arthritis and other rheumatic conditions and effect of patient's age on treatment choice. N. C. Med. J. 2011, 72, 432-438. [PubMed]

9. Wolfe, F.; Zhao, S.; Lane, N. Preference for nonsteroidal antiinflammatory drugs over acetaminophen by rheumatic disease patients: A survey of 1799 patients with osteoarthritis, rheumatoid arthritis, and fibromyalgia. Arthritis Rheumatol. 2000, 43, 378-385. [CrossRef]

10. Bhala, N.; Emberson, J.; Merhi, A.; Abramson, S.; Arber, N.; Baron, J.A.; Bombardier, C.; Cannon, C.; Farkouh, M.E.; FitzGerald, G.A.; et al. Vascular and upper gastrointestinal effects of non-steroidal anti-inflammatory drugs: Meta-analyses of individual participant data from randomised trials. Lancet 2013, 382, 769-779. [PubMed]

11. Fine, M. Quantifying the impact of nsaid-associated adverse events. Am. J. Manag. Care 2013, 19, s267-s272. [PubMed]

12. Serhan, C.N. Pro-resolving lipid mediators are leads for resolution physiology. Nature 2014, 510, 92-101. [CrossRef] [PubMed]

13. Calder, P.C. Marine omega-3 fatty acids and inflammatory processes: Effects, mechanisms and clinical relevance. Biochim. Biophys. Acta 2015, 1851, 469-484. [CrossRef] [PubMed]

14. Fortin, P.R.; Lew, R.A.; Liang, M.H.; Wright, E.A.; Beckett, L.A.; Chalmers, T.C.; Sperling, R.I. Validation of a meta-analysis: The effects of fish oil in rheumatoid arthritis. J. Clin. Epidemiol. 1995, 48, 1379-1390. [CrossRef]

15. Goldberg, R.J.; Katz, J. A meta-analysis of the analgesic effects of omega-3 polyunsaturated fatty acid supplementation for inflammatory joint pain. Pain 2007, 129, 210-223. [CrossRef] [PubMed]

16. Lee, Y.H.; Bae, S.C.; Song, G.G. Omega-3 polyunsaturated fatty acids and the treatment of rheumatoid arthritis: A meta-analysis. Arch. Med. Res. 2012, 43, 356-362. [CrossRef] [PubMed]

17. MacLean, C.H.; Mojica, W.A.; Morton, S.C.; Pencharz, J.; Hasenfeld Garland, R.; Tu, W.; Newberry, S.J.; Jungvig, L.K.; Grossman, J.; Khanna, P.; et al. Effects of omega-3 fatty acids on lipids and glycemic control in type ii diabetes and the metabolic syndrome and on inflammatory bowel disease, rheumatoid arthritis, renal disease, systemic lupus erythematosus, and osteoporosis. Evid. Rep. Technol. Assess. 2004, 89, 1-4.

18. Lane, K.; Derbyshire, E.; Li, W.; Brennan, C. Bioavailability and potential uses of vegetarian sources of omega-3 fatty acids: A review of the literature. Crit. Rev. Food Sci. Nutr. 2014, 54, 572-579. [CrossRef] [PubMed]

19. Gerster, H. Can adults adequately convert alpha-linolenic acid (18:3n-3) to eicosapentaenoic acid (20:5 n-3) and docosahexaenoic acid (22:6 n-3)? Int. J. Vitam. Nutr. Res. 1998, 68, 159-173. [PubMed]

20. Herman, C.J.; Allen, P.; Hunt, W.C.; Prasad, A.; Brady, T.J. Use of complementary therapies among primary care clinic patients with arthritis. Prev. Chronic Dis. 2004, 1, A12. [PubMed]

21. Christensen, R.; Bliddal, H.; Henriksen, M. Enhancing the reporting and transparency of rheumatology research: A guide to reporting guidelines. Arthritis Res. Ther. 2013, 15, 109. [CrossRef] [PubMed]

22. Liberati, A.; Altman, D.G.; Tetzlaff, J.; Mulrow, C.; Gotzsche, P.C.; Ioannidis, J.P.; Clarke, M.; Devereaux, P.J.; Kleijnen, J.; Moher, D. The prisma statement for reporting systematic reviews and meta-analyses of studies that evaluate health care interventions: Explanation and elaboration. Ann. Intern. Med. 2009, 151, W65-W94. [CrossRef] [PubMed]

23. Ghogomu, E.A.; Maxwell, L.J.; Buchbinder, R.; Rader, T.; Pardo, P.J.; Johnston, R.V.; Christensen, R.; Rutjes, A.W.; Winzenberg, T.M.; Singh, J.A.; et al. Updated method guidelines for cochrane musculoskeletal group systematic reviews and metaanalyses. J. Rheumatol. 2014, 41, 194-205. [CrossRef] [PubMed] 
24. Tugwell, P.S.; Maxwell, L.J.; Beaton, D.E.; Busse, J.W.; Christensen, R.; Conaghan, P.G.; Simon, L.S.; Terwee, C.; Tovey, D.; Wells, G.A.; et al. Deliberative dialogue on developing consensus on measurement and presentation of patient important outcomes in systematic reviews: A preconference meeting at omeract 12. J. Rheumatol. 2015. [CrossRef] [PubMed]

25. Maxwell, L.J.; Wells, G.A.; Simon, L.S.; Conaghan, P.G.; Grosskleg, S.; Scrivens, K.; Beaton, D.E.; Bingham, C.O., III; Busse, J.W.; Christensen, R.; et al. Current state of reporting pain outcomes in cochrane reviews of chronic musculoskeletal pain conditions and considerations for an omeract research agenda. J. Rheumatol. 2015. [CrossRef] [PubMed]

26. Juni, P.; Altman, D.G.; Egger, M. Systematic reviews in health care: Assessing the quality of controlled clinical trials. BMJ 2001, 323, 42-46. [CrossRef] [PubMed]

27. Higgins, J.P.; Altman, D.G.; Gotzsche, P.C.; Juni, P.; Moher, D.; Oxman, A.D.; Savovic, J.; Schulz, K.F.; Weeks, L.; Sterne, J.A. The cochrane collaboration's tool for assessing risk of bias in randomised trials. BMJ 2011, 343. [CrossRef] [PubMed]

28. Dwan, K.; Altman, D.G.; Arnaiz, J.A.; Bloom, J.; Chan, A.W.; Cronin, E.; Decullier, E.; Easterbrook, P.J.; Von, E.E.; Gamble, C.; et al. Systematic review of the empirical evidence of study publication bias and outcome reporting bias. PLoS ONE 2008, 3, e3081. [CrossRef] [PubMed]

29. Dwan, K.; Gamble, C.; Kolamunnage-Dona, R.; Mohammed, S.; Powell, C.; Williamson, P.R. Assessing the potential for outcome reporting bias in a review: A tutorial. Trials 2010, 11, 52. [CrossRef] [PubMed]

30. Hedges, L.V. Distribution theory for glass's estimator of effect size and related estimators. J. Educ. Behav. Stat. 1981, 6, 107-128. [CrossRef]

31. Normand, S.L. Meta-analysis: Formulating, evaluating, combining, and reporting. Stat. Med. 1999, 18, 321-359. [CrossRef]

32. Bliddal, H.; Christensen, R. The treatment and prevention of knee osteoarthritis: A tool for clinical decision-making. Expert Opin. Pharmacother. 2009, 10, 1793-1804. [CrossRef] [PubMed]

33. Tubach, F.; Ravaud, P.; Martin-Mola, E.; Awada, H.; Bellamy, N.; Bombardier, C.; Felson, D.T.; Hajjaj-Hassouni, N.; Hochberg, M.; Logeart, I.; et al. Minimum clinically important improvement and patient acceptable symptom state in pain and function in rheumatoid arthritis, ankylosing spondylitis, chronic back pain, hand osteoarthritis, and hip and knee osteoarthritis: Results from a prospective multinational study. Arthritis Care Res. 2012, 64, 1699-1707.

34. Higgins, J.P.; Thompson, S.G. Quantifying heterogeneity in a meta-analysis. Stat. Med. 2002, 21, 1539-1558. [CrossRef] [PubMed]

35. Higgins, J.P.; Thompson, S.G.; Deeks, J.J.; Altman, D.G. Measuring inconsistency in meta-analyses. BMJ 2003, 327, 557-560. [CrossRef] [PubMed]

36. Johansen, M.; Bahrt, H.; Altman, R.D.; Bartels, E.M.; Juhl, C.B.; Bliddal, H.; Lund, H.; Christensen, R. Exploring reasons for the observed inconsistent trial reports on intra-articular injections with hyaluronic acid in the treatment of osteoarthritis: Meta-regression analyses of randomized trials. Semin. Arthritis Rheum. 2016, 46, 34-48. [CrossRef] [PubMed]

37. Egger, M.; Davey, S.G.; Schneider, M.; Minder, C. Bias in meta-analysis detected by a simple, graphical test. BMJ 1997, 315, 629-634. [CrossRef] [PubMed]

38. R Development Core Team. A Language and Environment for Statistical Computing, 3.1.2, R Foundation for Statistical Computing: Vienna, Austria, 2014.

39. Guyatt, G.H.; Oxman, A.D.; Kunz, R.; Vist, G.E.; Falck-Ytter, Y.; Schunemann, H.J. What is "quality of evidence" and why is it important to clinicians? BMJ 2008, 336, 995-998. [CrossRef] [PubMed]

40. Darlington, L.G.; Ramsey, N.W. Olive oil for rheumatoid patients? Br. J. Rheumatol. 1987, 26, 129.

41. Tulleken, J.E.; Limburg, P.C.; Muskiet, F.A.J.; Kazemier, K.M.; Van Rijswijk, M.H. A Comparison between the Effects of Fish Oil Ethyl Esters and Fish Oil Triglycerides in Rheumatoid Arthritis, Long Chain Omega-3 Polyunsaturated Fatty Acids in Rheumatoid Arthritis. Thesis, Rijksuniversiteit Groningen, Groningen, The Netherlands, 1991.

42. Tulleken, J.E.; Limburg, P.C.; Wassenaar, W.; Van Rijswijk, M.H. Nonsteroidal Anti-Inflammatory Drug Demand during Fish Oil Treatment in Rheumatoid Arthritis, Long Chain Omega-3 Polyunsaturated Fatty Acids in Rheumatoid Arthritis. Thesis, Rijksuniversiteit Groningen, Groningen, The Netherlands, 1991.

43. Belch, J.J.F.; Ansell, D.; Madhok, R.; Sturrock, R.D. The effect of evening primrose oil epo and epo-fish oil combination on rheumatoid arthritis a double blind study. Br. J. Rheumatol. 1986, 25. 
44. Belch, J.J.; Ansell, D.; Madhok, R.; O’Dowd, A.; Sturrock, R.D. Effects of altering dietary essential fatty acids on requirements for non-steroidal anti-inflammatory drugs in patients with rheumatoid arthritis: A double blind placebo controlled study. Ann. Rheum. Dis. 1988, 47, 96-104. [CrossRef] [PubMed]

45. Cleland, L.G.; French, J.K.; Betts, W.H.; Murphy, G.A.; Elliott, M.J. Clinical and biochemical effects of dietary fish oil supplements in rheumatoid arthritis. J. Rheumatol. 1988, 15, 1471-1475. [PubMed]

46. Stammers, T.; Sibbald, B.; Freeling, P. Fish oil in osteoarthritis. Lancet 1989, 2, 503. [CrossRef]

47. Kremer, J.M.; Lawrence, D.A.; Jubiz, W.; DiGiacomo, R.; Rynes, R.; Bartholomew, L.E.; Sherman, M. Dietary fish oil and olive oil supplementation in patients with rheumatoid arthritis. Clinical and immunologic effects. Arthritis Rheum. 1990, 33, 810-820. [CrossRef] [PubMed]

48. Tulleken, J.E.; Limburg, P.C.; Muskiet, F.A.; van Rijswijk, M.H. Vitamin e status during dietary fish oil supplementation in rheumatoid arthritis. Arthritis Rheum. 1990, 33, 1416-1419. [CrossRef] [PubMed]

49. van der Tempel, H.; Tulleken, J.E.; Limburg, P.C.; Muskiet, F.A.; van Rijswijk, M.H. Effects of fish oil supplementation in rheumatoid arthritis. Ann. Rheum. Dis. 1990, 49, 76-80. [CrossRef] [PubMed]

50. Tulleken, J.E.; Limburg, P.; Vanrijswijk, M.H. Fish oil and plasma-fibrinogen. BMJ 1988, $297,615$.

51. Espersen, G.T.; Grunnet, N.; Lervang, H.H.; Nielsen, G.L.; Thomsen, B.S.; Faarvang, K.L.; Dyerberg, J.; Ernst, E. Decreased interleukin-1 beta levels in plasma from rheumatoid arthritis patients after dietary supplementation with n-3 polyunsaturated fatty acids. Clin. Rheumatol. 1992, 11, 393-395. [CrossRef] [PubMed]

52. Kjeldsen-Kragh, J.; Lund, J.A.; Riise, T.; Finnanger, B.; Haaland, K.; Finstad, R.; Mikkelsen, K.; Forre, O. Dietary omega-3 fatty acid supplementation and naproxen treatment in patients with rheumatoid arthritis. J. Rheumatol. 1992, 19, 1531-1536. [PubMed]

53. Kjeldsen-Kragh, J. Dietary treatment of rheumatoid arthritis. Scand. J. Rheumatol. 1996, 25, 63. [CrossRef]

54. Magarò, M.; Zoli, A.; Altomonte, L.; Mirone, L.; De Sole, P.; Di Mario, G.; De Leo, E. Effect of fish oil on neutrophil chemiluminescence induced by different stimuli in patients with rheumatoid arthritis. Ann. Rheum. Dis. 1992, 51, 877-880. [CrossRef] [PubMed]

55. Nielsen, G.L.; Faarvang, K.L.; Thomsen, B.S.; Teglbjaerg, K.L.; Jensen, L.T.; Hansen, T.M.; Lervang, H.H.; Schmidt, E.B.; Dyerberg, J.; Ernst, E. The effects of dietary supplementation with $n$-3 polyunsaturated fatty acids in patients with rheumatoid arthritis: A randomized, double blind trial. Eur. J. Clin. Invest. 1992, 22, 687-691. [CrossRef] [PubMed]

56. Faarvang, K.L.; Nielsen, G.L.; Thomsen, B.S.; Teglbjaerg, K.L.; Hansen, T.M.; Lervang, H.H.; Schmidt, E.B.; Dyerberg, J.; Ernst, E. Fish oils and rheumatoid arthritis. A randomized and double—blind study. Ugeskr. Laeger 1994, 156, 3495-3498. [PubMed]

57. Nielsen, G.L.; Faarvang, K.L.; Thomsen, B.S.; Teglibjaerg, K.L.; Ernst, E. Effects of supplementation with n-3 fatty acids on clinical disease variables in patients with rheumatoid arthritis. Eur. J. Clin. Invest. 1991, 21, 67.

58. Sköldstam, L.; Börjesson, O.; Kjällman, A.; Seiving, B.; Åkesson, B. Effect of six months of fish oil supplementation in stable rheumatoid arthritis. A double-blind, controlled study. Scand. J. Rheumatol. 1992, 21, 178-185. [CrossRef] [PubMed]

59. Stammers, T.; Sibbald, B.; Freeling, P. Efficacy of cod liver oil as an adjunct to non-steroidal anti-inflammatory drug treatment in the management of osteoarthritis in general practice. Ann. Rheum. Dis. 1992, 51, 128-129. [CrossRef] [PubMed]

60. Geusens, P.; Wouters, C.; Nijs, J.; Jiang, Y.; Dequeker, J. Long-term effect of omega-3 fatty acid supplementation in active rheumatoid arthritis. A 12-month, double-blind, controlled study. Arthritis Rheum. 1994, 37, 824-829. [CrossRef] [PubMed]

61. Kremer, J.M.; Lawrence, D.A.; Petrillo, G.F.; Litts, L.L.; Mullaly, P.M.; Rynes, R.I.; Stocker, R.P.; Parhami, N.; Greenstein, N.S.; Fuchs, B.R.; et al. Effects of high-dose fish oil on rheumatoid arthritis after stopping nonsteroidal antiinflammatory drugs. Clinical and immune correlates. Arthritis Rheum. 1995, 38, 1107-1114. [CrossRef] [PubMed]

62. Vargová, V.; Vesely, R.; Sasinka, M.; Torok, C. Will administration of omega-3 unsaturated fatty acids reduce the use of nonsteroidal antirheumatic agents in children with chronic juvenile arthritis? Cas. Lek. Cesk. 1998, 137, 651-653. [PubMed]

63. Volker, D.; Fitzgerald, P.; Major, G.; Garg, M. Efficacy of fish oil concentrate in the treatment of rheumatoid arthritis. J. Rheumatol. 2000, 27, 2343-2346. [PubMed] 
64. Adam, O.; Beringer, C.; Kless, T.; Lemmen, C.; Adam, A.; Wiseman, M.; Adam, P.; Klimmek, R.; Forth, W. Anti-inflammatory effects of a low arachidonic acid diet and fish oil in patients with rheumatoid arthritis. Rheumatol. Int. 2003, 23, 27-36. [PubMed]

65. Lau, C.S.; Chiu, P.K.Y.; Chu, E.M.Y.; Cheng, I.Y.W.; Tang, W.M.; Man, R.Y.K.; Halpern, G.M. Treatment of knee osteoarthritis with lyprinol, lipid extract of the green-lipped mussel-a double-blind placebo-controlled study. Prog. Nutr. 2004, 6, 17-31.

66. Sundrarjun, T.; Komindr, S.; Archararit, N.; Dahlan, W.; Puchaiwatananon, O.; Angthararak, S.; Udomsuppayakul, U.; Chuncharunee, S. Effects of n-3 fatty acids on serum interleukin-6, tumour necrosis factor-alpha and soluble tumour necrosis factor receptor p55 in active rheumatoid arthritis. J. Int. Med. Res. 2004, 32, 443-454. [CrossRef] [PubMed]

67. Berbert, A.A.; Kondo, C.R.; Almendra, C.L.; Matsuo, T.; Dichi, I. Supplementation of fish oil and olive oil in patients with rheumatoid arthritis. Nutrition 2005, 21, 131-136. [CrossRef] [PubMed]

68. Madland, T.M.; Bjorkkjaer, T.; Brunborg, L.A.; Froyland, L.; Berstad, A.; Brun, J.G. Subjective improvement in patients with psoriatic arthritis after short-term oral treatment with seal oil. A pilot study with double blind comparison to soy oil. J. Rheumatol. 2006, 33, 307-310. [PubMed]

69. Deutsch, L. Evaluation of the effect of Neptune krill oil on chronic inflammation and arthritic symptoms. J. Am. Coll. Nutr. 2007, 26, 39-48. [CrossRef] [PubMed]

70. Galarraga, B.; Ho, M.; Youssef, H.M.; Hill, A.; McMahon, H.; Hall, C.; Ogston, S.; Nuki, G.; Belch, J.J. Cod liver oil (n-3 fatty acids) as an non-steroidal anti-inflammatory drug sparing agent in rheumatoid arthritis. Rheumatology (Oxf., Engl.) 2008, 47, 665-669. [CrossRef] [PubMed]

71. Das Gupta, A.B.; Hossain, A.K.; Islam, M.H.; Dey, S.R.; Khan, A.L. Role of omega-3 fatty acid supplementation with indomethacin in suppression of disease activity in rheumatoid arthritis. Bangladesh Med. Res. Counc. Bull. 2009, 35, 63-68. [PubMed]

72. Gruenwald, J.; Petzold, E.; Busch, R.; Petzold, H.P.; Graubaum, H.J. Effect of glucosamine sulfate with or without omega-3 fatty acids in patients with osteoarthritis. Adv. Ther. 2009, 26, 858-871. [CrossRef] [PubMed]

73. Park, Y.; Lee, A.; Shim, S.C.; Lee, J.H.; Choe, J.Y.; Ahn, H.; Choi, C.B.; Sung, Y.K.; Bae, S.C. Effect of n-3 polyunsaturated fatty acid supplementation in patients with rheumatoid arthritis: A 16-week randomized, double-blind, placebo-controlled, parallel-design multicenter study in korea. J. Nutr. Biochem. 2013, 24, 1367-1372. [CrossRef] [PubMed]

74. Araújo, C.A.; Moraes-Fontes, M.F.; Santos, L.; Riso, N. Omega-3 fatty acids and mediterranean diet as complimentary therapies for rheumatoid arthritis. Arthritis Rheumatol. 2014, 66, S1050.

75. Stebbings, S.; Gray, A.; Schneiders, A.; Sansom, A. A novel green-lipped mussel lipid extract (biolex) for the treatment of pain in osteoarthritis of the hip and knee: A randomized double-blind, placebo controlled trial. Ann. Rheum. Dis. 2014, 73. [CrossRef]

76. Fu, Y.; Li, G.; Zhang, X.; Xing, G.; Hu, X.; Yang, L.; Li, D. Lipid extract from hard-shelled mussel (mytilus coruscus) improves clinical conditions of patients with rheumatoid arthritis: A randomized controlled trial. Nutrients 2015, 7, 625-645. [CrossRef]

77. Kremer, J.M.; Rynes, R.I.; Bartholomew, L.E.; Michalek, A.; Jubiz, W. A double-blinded placebo controlled crossover study of eicosapentaenoic-acid supplementation in active rheumatoid arthritis. Arthritis Rheum. 1986, 29, S11.

78. Hernández-Cruz, B.; Alcocer-Varela, J.; Cardiel, M.H. Omega-3 fatty acids supplementation in Mexican patients with rheumatoid arthritis with standard treatment. A blinded, randomized, placebo controlled, one year, clinical trial. Arthritis Rheum. 1998, 41, S155.

79. Kremer, J.; Larence, D.; Jubiz, W.; Digarcomo, R.; Rynes, R.; Bartholomew, L. Different doses of fish-oil fatty acid supplementation in rheumatoid arthritis ra a prospective double-blinded randomized study. Arthritis Rheum. 1988, 31, S303.

80. Kremer, J.M.; Lawrence, D.A.; Stocker, R.S.; Rynes, R.I.; Parhami, N.; Greenstein, N.S.; Petrillo, G.F.; Simon, L.S.; Robinson, D.W. Clinical, immunological and nsaid sparing effects of fish-oil (fo) in patients with active rheumatoid-arthritis (ra)-a long-term prospective randomized double-blind-study. Arthritis Rheum. 1993, 36, S56. 
81. Lau, C.S.; Morley, K.D.; Belch, J.J.F. Effects of fish-oil supplementation on nonsteroidal antiinflammatory drug requirement in patients with mild rheumatoid-arthritis-a double-blind placebo-controlled study. Br. J. Rheumatol. 1993, 32, 982-989. [CrossRef] [PubMed]

82. Lau, C.S.; McMahon, H.; Morley, K.D.; Belch, J.J.F. Effects of maxepa on non-steroidal anti-inflammatory drug usage in patients with mild rheumatoid arthritis. Br. J. Rheumatol. 1991, 30, 137.

83. Lau, C.S.; McLaren, M.; Belch, J.J. Effects of fish oil on plasma fibrinolysis in patients with mild rheumatoid arthritis. Clin. Exp. Rheumatol. 1995, 13, 87-90. [PubMed]

84. Kolahi, S.O.; Hejasi, J.A.; Mohtadinia, A. The effect of fish oil and vitamin e supplement over lipid peroxidation and antioxidant levels of plasma in patient with rheumatoid arthritis. Int. J. Rheum. Dis. 2010, 13, 108.

85. Kolahi, S.; Ghorbanihaghjo, A.; Alizadeh, S.; Rashtchizadeh, N.; Argani, H.; Khabazzi, A.R.; Hajialilo, M.; Bahreini, E. Fish oil supplementation decreases serum soluble receptor activator of nuclear factor-kappa $\mathrm{b}$ ligand/osteoprotegerin ratio in female patients with rheumatoid arthritis. Clin. Biochem. 2010, 43, 576-580. [CrossRef] [PubMed]

86. Ghorbanihaghjo, A.; Kolahi, S.; Seifirad, S.; Rashtchizadeh, N.; Argani, H.; Hajialilo, M.; Khabazi, A.; Alizadeh, S.; Bahreini, E. Effect of fish oil supplements on serum paraoxonase activity in female patients with rheumatoid arthritis: A double-blind randomized controlled trial. Arch. Iran. Med. 2012, 15, 549-552. [PubMed]

87. Kolahi, S.; Haghjoo, A.G. Effect of fish oil supplements on serum paraoxonase activity in patients with rheumatoid arthritis. Rheumatology (Oxf., Engl.) 2012, 51, i31.

88. Alizadeh, S.; Ghorbanihaghjo, A.; Kolahi, S.; Bahreini, E. Effect of omega-3 fatty acids supplementation on circulating osteoprotegerin ligand to osteoprotegerin ratio in female patients with rheumatoid arthritis. Clin. Biochem. 2011, 1, S142. [CrossRef]

89. Dawczynski, C.; Hackermeier, U.; Viehweger, M.; Stange, R.; Springer, M.; Jahreis, G. Incorporation of n-3 pufa and gamma-linolenic acid in blood lipids and red blood cell lipids together with their influence on disease activity in patients with chronic inflammatory arthritis-A randomized controlled human intervention trial. Lipids Health Dis. 2011, 10. [CrossRef] [PubMed]

90. Reed, G.W.; Leung, K.; Rossetti, R.G.; Vanbuskirk, S.; Sharp, J.T.; Zurier, R.B. Treatment of rheumatoid arthritis with marine and botanical oils: An 18-month, randomized, and double-blind trial. Evid. Based Complement. Altern. Med. 2014, 2014. [CrossRef] [PubMed]

91. Olendzki, B.C.; Leung, K.; Van Buskirk, S.; Reed, G.; Zurier, R.B. Treatment of rheumatoid arthritis with marine and botanical oils: Influence on serum lipids. Evid. Based Complement. Altern. Med. 2011, 2011, 827286. [CrossRef] [PubMed]

92. Yazdanpanah, P.; Mohamadi, H.; Sadeghi, H.; Ghaffarian Shirazi, H.; Arjmand, A. Survey of omega-3 effects on morning stiffness in osteoarthritis of the knee. Pain Pract. 2014, 14, 126.

93. Singh, G.; Chandra, R.K. Fish oils in rheumatoid arthritis. Ann. Intern. Med. 1988, 108, 904-905. [CrossRef] [PubMed]

94. Calder, P.C. Omega-3 polyunsaturated fatty acids and inflammatory processes: Nutrition or pharmacology? Br. J. Clin. Pharmacol. 2013, 75, 645-662. [CrossRef] [PubMed]

95. Stamp, L.K.; James, M.J.; Cleland, L.G. Diet and rheumatoid arthritis: A review of the literature. Semin. Arthritis Rheum. 2005, 35, 77-94. [CrossRef] [PubMed]

96. Villani, A.M.; Crotty, M.; Cleland, L.G.; James, M.J.; Fraser, R.J.; Cobiac, L.; Miller, M.D. Fish oil administration in older adults: Is there potential for adverse events? A systematic review of the literature. BMC Geriatr. 2013, 13. [CrossRef] [PubMed]

97. Cohen, J. Statistical Power Analysis for the Behavioral Sciences, 2nd ed.; Lawrence Erlbaum Associates: Hillsdale, NJ, USA, 1988.

(C) 2017 by the authors; licensee MDPI, Basel, Switzerland. This article is an open access article distributed under the terms and conditions of the Creative Commons Attribution (CC-BY) license (http://creativecommons.org/licenses/by/4.0/). 